\title{
Hydrophobic interaction chromatography for the characterization of monoclonal antibodies and related products
}

\author{
Szabolcs Fekete ${ }^{1 *}$, Jean-Luc Veuthey ${ }^{1}$, Alain Beck², Davy Guillarme ${ }^{1}$ \\ ${ }^{1}$ School of Pharmaceutical Sciences, University of Geneva, University of Lausanne, Boulevard d'Yvoy 20, 1211 \\ Geneva 4, Switzerland \\ ${ }^{2}$ Center of Immunology Pierre Fabre, 5 Avenue Napoléon III, BP 60497, 74160 Saint-Julien-en-Genevois, France \\ (www.cipf.com)
}

\section{Abstract}

Hydrophobic interaction chromatography $(\mathrm{HIC})$ is a historical strategy used for the analytical purification and characterization of proteins. Similarly to what can be done in reversed-phase liquid chromatography (RPLC), HIC is able to separate protein species based on their hydrophobicity, but using different conditions. Compared to RPLC, the main benefit of HIC is its ability to perform separations under non denaturing conditions (i.e. physiological pH conditions, ambient mobile phase temperature and no need for organic solvents) and so an orthogonal method. The goal of this review is to provide a general overview of theoretical and practical aspects of modern HIC applied for the characterization of therapeutic protein biopharmaceuticals including monoclonal antibodies (mAbs), antibody drug conjugates (ADCs) and bispecific antibodies (bsAbs). Therefore, method development approaches, state-of-the-art column technology, applications and future perspectives are described and critically discussed.

\section{Keywords:}

Hydrophobic interaction chromatography, antibody-drug-conjugate, therapeutic antibody, method development, columns 


\section{Introduction}

Monoclonal antibodies (mAbs) and related products such as antibody-drug conjugates (ADCs) and bispecific antibodies (bsAbs) are the fastest growing class of therapeutics [1]. Therefore, the need for analytical techniques applied for the detailed characterization of such biopharmaceuticals has increased drastically due to the growing number of approved therapeutic proteins and biosimilars (or follow-on-biologics) potentially entering the market. More than 60 lgGs and $\lg G$ derivatives have been approved so far for use in various indications such as cancers or inflammatory diseases [2]. ADCs become more and more popular, with two already approved drugs on the market (brentuximab vedotin - Adcetris, and ado-trastuzumab emtansine - Kadcyla) and more than 30 others in clinical trials [3,4]. ADCs are biochemotherapeutics constituted of a cytotoxic chemical drug covalently linked to a mAb. It combines the specificity of a mAb and the efficacy of the cytotoxic drug [5].

In general, the identity, heterogeneity, impurity content, and activity of each new batch of therapeutic proteins has to be thoroughly investigated before release. For the detailed characterization of mAbs and ADCs (and other related products), various chromatographic approaches such as reversed-phase (RPLC), size exclusion (SEC), ion exchange (IEX), hydrophobic interaction chromatography (HIC) or affinity chromatography are often applied [6]. Beside chromatographic techniques, capillary isoelectric focusing (cIEF), capillary zone electrophoresis (CZE), circular dichroism (CD), Fourier transform infrared spectroscopy (FT$\mathrm{IR})$, fluorescence spectrophotometry (FL), and mass spectrometry (MS) are also frequently used for the characterization. The goal of this multi-method strategy is to demonstrate the similarity between production batches by precisely characterizing the primary, secondary, and tertiary structure of the proteins $[7,8]$.

Among the several liquid chromatographic modes applied for the characterization of mAbs and $\mathrm{ADCs}, \mathrm{HIC}$ is the preferred technique for determining the relative hydrophobicity of mAbs and to separate the different populations of ADC molecules that differ in their number of drugs per antibody which are often known as DAR (drug-to-antibody ratio) species $[9,10]$. Positional isomers of DARs can also be separated by using high resolution HIC. The main 
advantage of HIC compared to other LC modes (e.g. RP) is that it is non-denaturating, so the native forms of the proteins are expected to be maintained. Moreover, the separated proteins can be collected for further activity measurements (such as cell based potency, receptor binding, cell proliferation assay, enzyme assay, functional ELISA...). HIC is also often used for protein purification based on the apparent hydrophobicity of impurities and is a valuable tool in downstream purification procedure $[11,12,13]$.

Previous reviews and book chapters have already presented earlier HIC works [12,13,14,15]. In this review, we focus on the possibilities of modern HIC applied for the characterization of therapeutic proteins (mAbs and related products), both from theoretical and practical points of view. Then, method development approaches, state-of-the-art column technology and applications are also reviewed and discussed.

\section{Theoretical aspects of HIC}

Since RPLC employs harsh conditions (i.e. mobile phase with acidic additive, organic modifier, and elevated temperature) that denaturate proteins, there was a need for alternative (milder) chromatographic conditions able to differentiate proteins based on their hydrophobicity. In this context, HIC was suggested as alternative [14], and its industrial significance has been growing. The main difference between HIC and RP is that proteins maintain their native structure with intra-molecular forces in $\mathrm{HIC}$ while they are denaturated in RP conditions. Figure 1 shows the chromatographic profiles of a reduced mAb obtained by $\mathrm{RP}$ and HIC. As can be seen, the original "Y" shape of the mAb is maintained in HIC conditions even if the disulphide bridges have been reduced. In contrast, the heavy- and light chains (Hc, Lc) are well separated in RP conditions. Despite the publication of many fundamental studies and retention models in the literature, the retention mechanism in HIC is often misunderstood and none of the proposed theories has received general acceptance.

Different interpretations and approaches such as hydrophobic interaction, hydrophobic effects, solvophobic theory, salting-out effect, dehydration of proteins or structural 
rearrangement of proteins are often confused. Here, we will try to clarify the various concepts and briefly summarize the different parameters affecting proteins retention in HIC.

[Figure 1 next here]

\subsection{Salting-out effect}

The concept of protein chromatography based on hydrophobic interactions was first described by Tiselius in 1948 [16]. He first used the term "salting-out chromatography" since salt solutions were applied as mobile phases. Salting-out effect is based on electrolyte / nonelectrolyte interaction (corresponding to mobile phase - protein interaction in $\mathrm{HIC}$ ), in which the nonelectrolyte becomes less soluble at high salt concentrations. In aqueous solutions, proteins fold and the hydrophobic amino acids usually form some protected hydrophobic areas, while hydrophilic amino acids form hydrogen bonds with the surrounding water. If the hydrophilic surface of the protein is large enough, then the protein can be dissolved in water. When adding salts, water molecules will solvate predominantly salt ions. Therefore, the number of water molecules available to interact with the hydrophilic part (charged) of the protein will decrease. Under these conditions, the protein-protein intermolecular interactions become stronger due to the decreased amount of surrounding water molecules. At the end, the protein molecules can associate by forming hydrophobic interactions with each other (aggregation).

Later on, this separation mode was called as "hydrophobic chromatography" or "hydrophobic affinity chromatography" [17]. The name "hydrophobic interaction chromatography" was introduced by Hjertén [11], but he also named this mode as "salt mediated separation of proteins". In 1986, Porath suggested "salt-promoted adsorption" or "salt-promoted adsorption chromatography" (SPAC) as alternative expressions for HIC [18].

\subsection{Hydrophobic effects, formation of cavity}

The hydrophobic effect is generally defined as an interaction of nonpolar substances or moieties of the molecules with water that is responsible for their low solubility $[19,20]$. On the 
other hand, the term "hydrophobic interactions" has also been used to describe the forces resulting in the association of nonpolar molecules or the binding of hydrophobic moieties in aqueous solutions [21]. Hydrophobicity generally means the repulsion between a non-polar moiety of the protein and of the polar aqueous environment of water [12]. The structure of water is highly ordered and stabilized by dipole-dipole interactions over a 3-dimensional structure, characterized by the high surface tension of water $\left(\sim 72 \mathrm{mN} / \mathrm{m}\right.$ at $\left.25^{\circ} \mathrm{C}\right)$ [22]. Each oxygen atom has four hydrogens as neighbors in a tetraeder configuration, and each hydrogen atom forms a bridge between two oxygen atoms (through covalent or hydrogen bonds). When dissolving hydrophobic moieties of a protein in an aqueous system, the neighboring water molecules have to be separated from each other in order to form a cavity for the protein [22]. This requires the investment of energy, which corresponds to the surface of the cavity multiplied by the surface tension. If two or more partners are associated, their hydrophobic contact surface area is reduced, and energy is released. The amount of energy is proportional to the size of the hydrophobic contact surface area of the protein. In other words, the interaction between two or more hydrophobic molecules in aqueous solutions takes place spontaneously and is mainly driven by the entropy change [23,24,25]. Frank and Evans reported that the large entropic effect arises from an orientation of the water molecules at the cavity around the nonpolar solute molecule (protein) $[26,27]$. Further studies indeed showed that the heat capacity change in such transfer-into-water processes is determined by the change in the water-accessible nonpolar surface area of the molecule $[28,29]$. At first, it was assumed that hydrophobic interactions involve only entropic effects. However, Baldwin has shown that hydrophobic interactions are entropy driven at low temperatures, but enthalpy driven at elevated temperatures, when the heat capacity change remains constant in the range of experimental temperature [30]. Such model experiments provided the basis of a more detailed understanding of the influence of temperature on hydrophobic interactions and hydrophobic effect.

\subsection{Solvophobic theory}


In general, the solvophobic theory explains the interactions between polar solvent (aqueous mobile phase) and less polar solute (protein). Due to $\mathrm{H}$-bonding and other polar interactions, strong cohesive forces exist between the solvent molecules and provide a strongly structured order for the solvent. Therefore, less polar solutes tend to be insoluble due to the strong solvent-solvent binding interactions. The retention in RPLC is often explained by the solvophobic theory. According to this, the solute molecules stick to the surface of the stationary phase due to their rejection form the solvent and their affinity for the hydrophobic stationary phase. So, the retention is partly explained by the interactions between the solute and stationary phase and partly by the rejection of solute from the mobile phase solvent.

Horváth et al developed the basis for describing retention mechanisms in RPLC, employing the framework of the solvophobic theory [31]. A simplified mass balance equation was introduced and led to an expression of the different free energy contributions to the overall retention process. It was shown that the structural forces of $\mathrm{H}$-bond interlinked water molecules represent an energetically low state of the water structure. In contrast, in the neighborhood of the stationary phase alkyl-chains, the water (or aqueous solvent) is under an energetically "excited" state as it has no contact with neighboring water molecules. One way to return to an energetically low state is an enforced association among the alkyl-chains, and another way is to combine the alkyl-ligand and hydrophobic solute to form an association complex. Horváth called the latter one as the "reversed phase retention process". The disappearing contact surface area multiplied by the surface tension represents the released energy upon association of the hydrophobic ligand with the analyte molecule, suggesting that the retention is an exothermic process. It was also found that the dominant term in the free energy equation was the so called "cavity term", which expresses the large energy required to separate neighboring water molecules in order to form a cavity in the aqueous mobile phase around the alkyl chains. More interested readers can find details in the review of Molnár on solvophobic theory [22].

A comprehensive treatment of the salting-out of proteins and the salt effect on HIC retention in the absence of specific salt binding is based on the adaptation made by Horváth and co- 
workers [32]. Within this theory, the retention free energy is expressed by the surface tension of aqueous salt solutions and the hydrophobic contact surface area between the solute and the ligand upon binding. By accounting for the effect of salt concentration on the mobile phase surface tension, the magnitude of solute retention has been expressed in terms of the molar salt concentration in HIC $[32,33]$. The theory predicts that for sufficiently high salt concentrations - where the retention is governed predominantly by hydrophobic interactions the retention increases with both the molar salt concentration (in the mobile phase) and the size of the solute (protein) - or its hydrophobic moiety.

Figure 2 shows a schematic view of protein self-association and ligand binding under typical HIC conditions.

[Figure 2 next here]

\subsection{Salting-in effect}

In contrast with the "salting-out" effect, when adding salts having divalent cations and univalent anions such as $\mathrm{MgCl}_{2}$ or $\mathrm{CaCl}_{2}$, some specific interactions can occur with proteins $[34,35]$. Due to these interactions, such salts do not enhance protein retention as much as expected from the increase of the surface tension and can even reduce retention. This is in agreement with the observation that these salts increase protein solubility (salting-in properties) [14]. The phenomenon was explained by the interaction of the salt to the protein surface. Salts exhibiting this behavior were called "chaotropic salts" [36]. Timasheff and coworkers studied the specific interaction of proteins in HIC with a number of solvent components, including salts with univalent and divalent cations and developed the theoretical framework $[36,37,38,39,40,41]$. In routine HIC separations the use of chaotropic salts is not common.

\section{Method development in HIC}

Practical method development aspects are rarely reported in the literature for HIC [42]. In this section, the most important parameters affecting selectivity and retention in HIC are 
described, and the possibility of automated method development is discussed. Very recently, a generic method development approach for mAbs and ADCs' DARs separations was proposed and will be considered as a reference example $[43,44]$.

\subsection{The impact of stationary phase}

In HIC, mildly hydrophobic stationary phases are used in most cases (much less hydrophobic than in RPLC). Most common ligands include relatively short n-alkyls (butyl, hexyl, octyl), phenyl or ether, which are linked to the silica or polymeric material through several coupling approaches $[13,45,46]$. The hydrophobicity of the stationary phase increases with the length of the alkyl-chain, but the loading capacity may decrease. The strength of hydrophobic interactions between the protein and stationary phase ligand can be easily controlled by the ligand density (loading) [13]. The relationship between ligand density and protein retention is dependent on the size of the protein, although the protein surface hydrophobicity should be considered as the most affecting factor [47]. It was found that the major contributor to the increase of adsorption enthalpies with ligand density is the heat required for the dehydration process of the protein and the adsorbent, as well as the structural rearrangement of the protein [48]. Therefore, ligand density is an important tool that can be modified to obtain distinct types of stationary phases with different selectivity and binding capacities. Aromatic ligands can provide some additional $\pi-\pi$ interactions which can strengthen the interaction and therefore increase retention [49].

In the past $10-20$ years, the number of commercially available analytical scale HIC stationary phases was quite limited, but this number has recently grown exponentially, thanks to the recent developments in column technology and strong interest for protein biopharmaceuticals. The first type of HIC phases were based on polysaccharide gels (e.g. agarose, cellulose, dextran) but now, analytical scale HIC columns are based mostly on silica or polymer particles [50]. Both porous and non-porous particles are available. Obviously nonporous materials provide much higher efficiency for proteins due to their reduced mass transfer resistance. Highly cross-linked non-porous poly(styrene-divinylbenzene) (PS/DVB) 
and polymethacrylate-based particles are also frequently used. Some recent HIC stationary phases packed with non-porous polymer particles are TSKgel Butyl-NPR (Tosoh), HIC Phenyl PH-814 (Shodex), Proteomix HIC Butyl, -Ethyl, -Propyl, -Phenyl (Sepax). There are also some porous polymethacrylate-based phases such as TSKgel Ether-5PW and TSKgel Phenyl-5PW (Tosoh). The latest silica-based porous particles are available with alkyl-amide or butyl phases and include MAbPac HIC 10 (Thermo), MAbPac HIC-Butyl (Thermo), MAbPac HIC 20 (Thermo) or Protein-Pak Hi Res HIC (Waters) [50]. State-of-the-art columns can now withstand pressure drop of up to 100-400 bar and columns are typically packed with 10, 7, 5, 3 and $2.5 \mu \mathrm{m}$ particles. Modern HIC columns have recently been characterized by mean of their hydrophobicity, efficiency, selectivity and peak capacity, when applied for mAb and ADC analysis [44]. Even if those columns are dedicated for mAb and ADC separations, some of them are clearly too hydrophobic for the elution of the most hydrophobic DAR species (DAR6 and DAR8) of ADC brentuximab vedotin [44].

Column diameters (I.D.) between 2 and $8 \mathrm{~mm}$ are available but $4.6 \mathrm{~mm}$ I.D. columns are the most widely used in current HIC applications. By varying the column length, the separation power and analysis time can be adjusted. On $4.6 \mathrm{~mm}$ I.D. columns, a flow rate of $0.6-1.0$ $\mathrm{mL} / \mathrm{min}$ is applied and the analysis times usually range between 10 and 20 minutes for $10 \mathrm{~cm}$ long columns [50].

Figure 3 shows the impact of stationary phase nature on selectivity and retention when separating a mixture of five commercial therapeutic mAbs. The same ammonium acetate gradient was applied on all the three columns $(100 \times 4.6 \mathrm{~mm})$ and the other conditions were identical. As illustrated, the Waters Protein Pak column provided the lowest retention, while the Thermo HIC 10 column gave the highest retention. Selectivity was also slightly altered, but the most important differences were observed for peak broadening and tailing.

[Figure 3 next here]

3.2. The impact of mobile phase composition 
The selection of mobile phase and operating conditions are mostly based on subjective and historical references. As an example, in most HIC applications, butyl phases are almost exclusively operated in ammonium sulfate buffer. However, all the salts having salting out properties (and appropriate solubility) can be considered as potential buffer components for HIC separations. Mobile phase $\mathrm{pH}$, salt concentration and type, as well as organic modifiers can also impact selectivity and retention in HIC.

\subsubsection{Impact of the salt nature and concentration}

In HIC practice, an inverse salt gradient is applied to elute proteins from a mildly hydrophobic stationary phase. By using gradient mode, proteins possessing a wide range of hydrophobicity can be eluted. Historically, 1.5 - $2 \mathrm{M}$ ammonium-sulfate aqueous solution is often used as mobile phase "A", but it is important to keep in mind that various salts can be applied. The influence of different salts on hydrophobic interactions follows the lyotropic (Hoffmeister) series for the precipitation of proteins from aqueous solutions [51]. In this series, salts are ranked in order of their salting-out effect. The salts at the beginning of this series promote hydrophobic interactions and protein precipitation. They are often called as antichaotropic salts and include anions such as phosphate, sulfate, acetate or chloride and cations such as ammonium, potassium or sodium. In practice, sodium or ammonium sulfate effectively promote stationary phase-protein interactions and have a stabilizing influence on protein structure. Hence, the most commonly used salts are ammonium sulfate, sodium sulfate as well as sodium chloride and ammonium acetate. Alternative salt systems were also studied and it was found that sodium acetate increased the retention of hydrophobic proteins, while it decreased retention of the hydrophilic ones [52]. In other early studies, sodium citrate was found also to be a good candidate, but was left out because of solubility issues $[53,54]$. It has also been demonstrated that salt nature affects differently the retention on different stationary phases. It can both increase and decrease the retention of hydrophobic and hydrophilic proteins, respectively [53,54]. The above results also imply that 
the effect of a salt cannot be predicted in advance but should always be determined experimentally as an early step of method development [42].

Beside the salt type, the other parameter for varying HIC retention is the salt concentration. Depending on the lyotropic strength of the various salts, different concentrations are required to maintain the same salting-out effect. Stronger salts are efficient in 1-1.5 M concentration, but weaker salts require higher concentration $(3-5 \mathrm{M})$ to maintain the same retention. On the other hand, peak widths also vary with salt concentration, since it impacts the concentration gradient (steepness) and therefore the gradient band focusing effect. A systematic study showed the possibility to combine different salts (binary and ternary salt systems) to modify selectivity and retention in HIC [42]. In this phase system optimization concept, the experimental design was based on gradient experiments performed on three different columns and with three different buffers (salts).

A recent study demonstrated that for ADC DAR separations, similar selectivity can be achieved with any type of salts, provided that its lyotropic strength is corrected on a given stationary phase $[43,44]$. Equivalent molarities of the different salt systems were determined for ADC separations on various columns. As example, on a Thermo HIC 10 column, $1 \mathrm{M}$ ammonium sulfate was equivalent to $2.2 \mathrm{M}$ sodium acetate, $2.6 \mathrm{M}$ sodium chloride and $3.3 \mathrm{M}$ ammonium acetate. Similarly, 2.4 M sodium acetate, $3.9 \mathrm{M}$ sodium chloride and $4.2 \mathrm{M}$ ammonium acetate could replace $1 \mathrm{M}$ ammonium sulfate on a Waters Protein-Pak column. A Tosoh TSKgel butyl NPR column showed similar behavior to the Protein-Pak (in terms of salt interchangeability). On a Tosoh TSK gel ether column, significantly higher salt concentration was required to maintain the same selectivity and retention. By using this column, $1 \mathrm{M}$ ammonium sulfate was equivalent to $3.9 \mathrm{M}$ sodium acetate, and could be replaced by $5.2 \mathrm{M}$ and 5.4 M sodium chloride and sodium acetate, respectively.

Hydrophobicity indexes were also derived for various mAbs and ADC DARs on several stationary phases using various salt systems [44]. For mAbs, the hydrophobicity indexes varied significantly within the different phase systems (the combination of the salt and stationary phase), but the elution order remained identical in each condition. Hydrophobicity 
indexes were found to be dependent on the stationary phase for the same salt system. This suggests that mAbs retention (and therefore selectivity) strongly depends on the stationary phase. The selectivity of mAb separations performed on one given column can also be tuned by changing the salt type.

\subsubsection{Impact of $\mathrm{pH}$}

The effect of $\mathrm{pH}$ in HIC is not straightforward $[55,56,57,58]$. In most cases, a pH increase, reduces the hydrophobic interactions between proteins and the hydrophobic ligands of the stationary phase, due to the increased hydrophilicity promoted by the change in the protein charge. On the other hand, a pH decrease can result in an apparent increase of hydrophobic interactions. Hjertén et. al. found that the retention of various test proteins changed more drastically at $\mathrm{pH}$ values above 8.5 and/or below 5 than in the range $\mathrm{pH} 5-8.5$ [59]. Obviously the shift in retention caused by the $\mathrm{pH}$ is protein dependent (the $\mathrm{p} /$ and the number of charged amino acid residues have an impact). Each protein could have different behaviour when changing the $\mathrm{pH}$, and therefore mobile phase $\mathrm{pH}$ could be considered as an adiditonal parameter for tuning selectivity and retention in HIC. However, since HIC is a nondenaturating chromatographic mode, it is recommended to use a $\mathrm{pH}$ close to physiological conditions, when analyzing mAbs and ADCs.

A recent work described the impact of mobile phase $\mathrm{pH}$ on $\mathrm{mAb}$ and $\mathrm{ADC}$ DARs retention and selectivity in the physiological $\mathrm{pH}$ range from 6.3 to 7 [43]. In this narrow $\mathrm{pH}$ range, no difference or shift in retention was observed for intact mAbs and ADC species. Such a behaviour was expected since mAbs and ADCs possess relatively high $\mathrm{p} /$ values (typically between 8 and 9). These findings suggest that in the physiological $\mathrm{pH}$ range, the methods are expected to be robust for $\mathrm{pH}$ variation and $\mathrm{pH}$ has a minor impact on selectivity and retention.

\subsubsection{Temperature effects}


The effects of temperature on solute retention in liquid chromatography are often expressed with the Gibbs free energy (van't Hoff equation). In most chromatographic modes, with "regular" analytes, the retention decreases with increasing temperature and a linear trend is observed when plotting log $(k)$ versus $1 / \mathrm{T}$. In HIC, the effect of temperature is not trivial and different (contrasting) behaviour were reported.

The retention of proteins in $\mathrm{HIC}$ is often increased with temperature, and this effect has been attributed to the enhanced magnitude of hydrophobic interactions resulting from temperatureinduced conformational changes of proteins or to the concomitant increase in the hydrophobic contact area upon binding to the stationary phase $[60,61]$. Horváth and coworkers determined the individual relative contributions of enthalpy and entropy to the free energy change upon adsorption as a function of temperature [14]. Based on the Kirchoff heat capacity change, they proposed a model called "the logarithmic equation" that describes the logarithmic retention factor as a function of temperature [62]. They experimentally confirmed that enthalpy and entropy changes were large and positive at low temperatures, then decreased with increasing temperature, and finally became negative at high temperatures. Measurements also confirmed the existence of certain exo-thermodynamic relationships, such as enthalpy - entropy compensation and molecular area correlations.

\subsubsection{Impact of organic modifier}

Adding a low proportion of water-miscible alcohols to the mobile phase results in weakening the protein-ligand interactions in $\mathrm{HIC}$, leading to a retention decrease [55]. The non-polar parts of alcohols (often isopropanol) compete effectively with the bound proteins for the adsorption sites on the HIC stationary phase causing the displacement of the protein. Alcohols also decrease the surface tension of mobile phase, thus weakening the hydrophobic interactions to give a subsequent dissociation of the ligand-solute complex [55]. Although the addition of organic modifiers to the mobile phase can be useful to alter the retention of proteins, there is a risk that proteins could be denatured or inactivated. Therefore in HIC conditions, only limited amount of alcohols can be added (e.g. $<15 \%$ ). 
Addition of organic modifiers, such as isopropanol, is often cited as an advantageous parameter in HIC method development for decreasing the retention of too hydrophobic compounds and also to adjust selectivity [56].

In practice, HIC separations are performed by developing a reverse salt gradient. If organic modifiers have to be used, it should only be added into the mobile phase " $\mathrm{B}$ ". Therefore, when developing the reverse salt gradient, an organic modifier gradient is also performed (e.g. increase of isopropanol content during the gradient program). Addition of organic modifiers to the mobile phase "A" should be avoided since the solubility in such a large concentration of salts is too limited and precipitation can occur.

The impact of isopropanol content in mobile phase "B" on the retention of both mAbs and ADC DARs was evaluated in a systematic way, in a recent work [43]. Generic HIC gradient was applied and the isopropanol content of mobile phase " $\mathrm{B}$ " was varied between 0 and $15 \%$. Figure 4 shows the change in retention and selectivity of intact mAbs when changing the isopropanol content in mobile phase "B". The chromatograms clearly show the reduction of retention and change of selectivity between peaks (see for example the elution order change of peaks 3 and 4). Based on these observations, the isopropanol content of the mobile phase may be a valuable parameter to tune selectivity of mAbs separation in HIC.

[Figure 4 next here]

However, for a cysteine linked IgG1 type ADC (Brentuximab Vedotin), a different behaviour was observed. As expected, a retention decrease was observed for DAR0 and DAR2 species, but surprisingly the retention of DAR 4, DAR6 and DAR8 increased with the isopropanol concentration. Moreover at $9 \%$ of isopropanol, the DAR8 cannot be eluted from the column and the selectivity between DAR4 and DAR6 decreased. Some possible explanations for this specific retention behaviour are i) a shift in mobile phase $\mathrm{pH}$ and proteins $\mathrm{p} /$ when adding isopropanol, taking into account that the $\mathrm{pH}$ shift could be different depending on the amount of salts that is continuously changing during the gradient; ii) Conformational changes of ADCs in presence of alcohols. To conclude on the addition of isopropanol for ADC analysis as a parameter of method development, it does not seem to be 
useful since it increases the retention of the most hydrophobic ADC DAR species and does not permit the elution of DAR6 and DAR8 from the column [43].

\subsection{Linear Solvent Strength model}

The Linear Solvent Strength (LSS) model is frequently applied in various modes of liquid chromatography to describe the relation between the solute retention and experimental conditions (i.e. gradient slope or mobile phase composition) [63]. The dependence of the retention on the mobile phase salt concentration (ionic strength) determines the applicability of the LSS model for gradient elution in HIC [64].

In isocratic elution mode, a logarithmic retention factor $(\log (k))$ vs. salt concentration (c) function typically shows linear behavior and can be expressed as:

$\log k=\log k_{0}+S \cdot c$

where $k$ is the retention factor $\left(k=\left(t_{r}-t_{0}\right) / t_{0}, t_{r}\right.$ refers to the solute retention time and $t_{0}$ refers to column dead time), $k_{0}$ corresponds to the retention factor observed in mobile phase containing no salt, $c$ is the salt concentration and $S$ is the gradient steepness.

According to the LSS theory, the retention time of a solute can be calculated in HIC conditions for any gradient using the following equation:

$t_{r}=\frac{t_{G}}{S \cdot \Delta c} \log \left(\frac{2.303 \cdot S \cdot \Delta c \cdot t_{0} \cdot k_{0}}{t_{G}}+1\right)+t_{0}+t_{d}$

where $t_{G}$ is the gradient time (duration) and $t_{d}$ is the system dwell time (gradient delay). It was experimentally shown by Szepesy and Karger that the retention of some common proteins in HIC gradient mode is in quantitative agreement with the LSS model $[34,42]$. Then, this model should enable retention prediction and modeling in HIC $[43,44]$.

\subsection{Computer assisted method optimization, retention modelling}

Szepesy and co-workers suggested, that the first step - for systematic method development in HIC - should be the selection of an appropriate phase system (the stationary and mobile 
phase) which is suitable for the required separation (provides adequate retention and selectivity) [42]. They developed a protocol for phase system characterization based on gradient experiments performed with three different gradient steepness performed on three columns and using three salts as mobile phase constituent. It was found that the hydrophobicity index was the most useful characteristic to compare the different phase systems. This phase system optimization approach was recently updated for therapeutic mAbs and ADCs using the latest column technology and supported by computer modeling [44].

After finding the appropriate phase system (column, salt type and concentration), the next step is the fine tuning of method parameters such as the (1) gradient steepness, (2) temperature (3) organic modifier and perhaps (4) mobile phase $\mathrm{pH}$. Above all, clearly the gradient steepness (program) plays the major role for tuning retention and selectivity and should be optimized both for mAbs and ADCs. Temperature can also be a useful parameter, but it can only be optimized in a very narrow range (e.g. between 20 and $40 \stackrel{\circ}{\circ}$ ) in order to maintain physiological-like conditions. The possible addition of organic modifier to the mobile phase in HIC, was found to be particularly useful for mAbs separations, as some elution order changes between peaks were noticed. On the contrary, when dealing with the analysis of ADC DAR species in HIC, the addition of isopropanol was not beneficial since the most hydrophobic species (DAR6 and DAR8) cannot be eluted in presence of $\sim 10 \%$ isopropanol. Finally, within a narrow pH range (eg. $6<\mathrm{pH}<7$ ), no important change in retention was observed for mAbs and ADC species, therefore this parameter can be considered for optimization only in a few specific cases.

Optimization software packages generally employ linear models for the determination of the unknown coefficients of the retention models, for the simultaneous optimization of two or three variables. Software implements an interpretive approach, where the retention behaviour is modelled using experimental information from initial runs, and the retention times at other conditions are predicted in a selected experimental domain. This allows 
calculating the critical resolution, and accordingly, determining the optimal separation $[65,66]$. Two or three dimensional models can be built up in such software (e.g. DryLab 4).

Currently, columns of $100 \times 4.6 \mathrm{~mm}$ are used in HIC practice. Considering this column dimension, it was suggested to perform $t_{g 1}=10$ and $t_{g 2}=30$ min long linear gradients at a flow rate of $0.6 \mathrm{~mL} / \mathrm{min}$. Then gradient program can be optimized and retention times, selectivities or resolutions can be predicted for any gradient condition, since retention behaviour followed the LSS model $[43,44]$. Beside this one dimensional retention model, the optimization of temperature and organic modifier content can also be useful for the separation of mAbs and related products. Linear models accurately describe the retention for both variables $[43,44]$. Therefore, gradient steepness can be combined with temperature or organic modifier in two dimensional retention models. Alternatively, a three dimensional model can also be build up by the combination of all the three variables. Regarding temperature, it is suggested to work at two levels as $T_{1}=20{ }^{\circ} \mathrm{C}$ and $T_{2}=40{ }^{\circ} \mathrm{C}$. Organic modifier levels can be varied as $c_{\text {org } 1}=0$ and $c_{\text {org } 2}=10 \%$ in mobile phase "B". This two dimensional model requires 4 initial experimental runs $\left(2 t_{g} \times 2 T\right.$ or $\left.2 t_{g} \times 2 c_{\text {org }}\right)$, while the three dimensional retention model necessitates 8 initial experiments $\left(2 t_{g} \times 2 T \times 2\right.$ corg). Following the execution of the input experimental runs, peak tracking has to be performed (alignment of the peaks under the different conditions). Peak tracking can be carried out on the basis of peak areas and/or individual injections of the mAbs or ADC species, when possible. Finally, the optimization can be carried out on the basis of the created resolution map. In the resolution map, the smallest resolution $(R s)$ value of any two critical peaks in the chromatogram is plotted as a function of the experimental parameters. By applying this approach, the average relative error of retention time prediction is not higher than $1.0 \%$ and method optimization can be performed within a few hours [43,44]. Figure 5 shows the schematic view of systematic method development in HIC for mAb or ADC DAR separations.

[Figure 5 next here]

\section{Application of HIC for protein separations}


HIC is a widely used approach for protein purifications $[15,67,68,69,70]$. In this review, we focus only on analytical scale separations and mAbs/ADCs applications. In this field, the main applications are 1) the monitoring of various post-translational modifications, 2) the determination of ADCs' average DAR and DAR distribution, 3) the determination of mAbs or ADCs hydrophobicity and 4) the determination of heterodimerization efficiency of bispecific antibodies. Recent examples of these different applications are reviewed below.

\subsection{Monitoring post translational modifications}

Even if RPLC is today the gold standard for analytical purposes, due to the revolutionary improvements in RP column technology, HIC remains an interesting and complementary strategy, especially for $\mathrm{ADC}$ and $\mathrm{mAb}$ characterization. Various types of post translational modifications of mAbs, ADCs and related proteins can be monitored in HIC, including degradations, fragmentation, misfolding, oxidation, carboxy terminal heterogeneity, aspartic acid isomerization, unpaired cysteins [10].

Oxidation of exposed amino acid side chains such as tryptophan or methionine is commonly observed in recombinant therapeutic proteins. Monitoring oxidation is of particular importance when residues undergoing this type of degradation. Only few methods are available to monitor such modifications (HIC, IEX, RPLC). In a recent publication, HIC was described as a chromatographic method able to monitor the oxidation of tryptophan residues located in recombinant mAb [71]. The elution profile presented two well-resolved pre-peaks from the main isoform. The method used two $100 \mathrm{~mm}$ long HIC columns coupled in series to improve resolution. Peaks were eluted via reverse gradient of ammonium sulfate buffered at pH 5.5 and supplemented with $10 \%$ acetonitrile as organic modifier.

Balland et al. developed a HIC method to separate and characterize populations of mAbs resulting from variable $\mathrm{N}$ - and C-terminal processing, stressed-induced covalent modifications and conformationally altered populations present in the drug product [72]. The potential of HIC was studied to characterize heterogeneity in the intact molecule and the Fab and Fc sub-domains resulting from papain cleavage. This study demonstrated the utility of 
HIC for assessing heterogeneity, stability and, in some cases, potency of mAbs. Figure 6 shows an example on the separation of modified mAb species when storing at $40 \stackrel{\circ}{\mathrm{C}}$ for 12 weeks. According to the authors, the pre-peaks correspond to Asp $\rightarrow$ iso-Asp modifications in the light chain.

[Figure 6 next here]

HIC was also applied to monitor levels of unpaired cysteine residues in the Fab of an IgG1 mAb [73]. A Tosoh TSKgel phenyl-5PW column was used in combination with a gradient of ammonium sulfate buffered with TRIS- $\mathrm{HCl}$ at $\mathrm{pH} 7.5$, to separate omalizumab Fab with unpaired Cys ${ }^{22}$ and Cys ${ }^{96}$.

HIC was also successfully implemented for purity testing of the fusion protein etanercept [74]. Early eluting pre-peaks of etanercept contained truncated variants. Post-peaks included dimers and etanercept associated with Chinese hamster ovary $(\mathrm{CHO})$ host cell proteins. This method was also used to compare commercial and biosimilar products.

\subsection{Calculation of Antibody-Drug Conjugates' average drug to antibody ratio (DAR)}

HIC is widely applied to separate DAR species of thiol conjugated (cysteine linked) ADCs. Thiol conjugates are produced by the partial reduction of disulphide bridges and followed by the conjugation with a drug linker, resulting in a heterogeneous population that differ with respect to the site of conjugation and the number of drugs per antibody (DAR) [9]. Both IgG1 and IgG2 type antibodies are used to create ADCs. As IgG1s and IgG2s differ in the number of disulfide bridges (4 and 6, respectively) and molecular conformation, each subclass can lead to unique combinations of possible conjugation sites. The drug-loading distribution and conjugation sites of ADCs have been reported to influence pharmacokinetic, toxicity, clearance and therapeutic index $[75,76,77]$. Therefore it is important to determine the average DAR and distribution of the different populations. One of the most important quality attributes of an ADC is the average number of drugs that are conjugated, because this determines the amount of "payload" that can be delivered to the tumor cell. A fully conjugated $\lg \mathrm{G} 1 \mathrm{ADC}$ has a maximum DAR of 8 , while an $\lg \mathrm{G} 2 \mathrm{ADC}$ has a maximum DAR of 12 
(because of the two additional disulphide bridges). IgG1 ADCs are composed of a heterogeneous mixture of $0,2,4,6$, and 8 DARs and IgG2 ADCs of $0,2,4,6,8,10$ and 12 DARs [78]. An odd number (e.g. DAR 1 or DAR 3) of conjugated drug is typically indicative of incomplete conjugation or degradation, and mostly observed in very small amount [9]. DAR 0 corresponds to the naked $\mathrm{mAb}$ and is considered as an impurity of the ADC. Figure $7 \mathrm{~A}$ illustrates the possible positional isomers of $\lg \mathrm{G} 1$ and $\lg \mathrm{G} 2$ types thiol conjugations and figure $7 \mathrm{~B}$ and $\mathrm{C}$ show representative HIC chromatograms of both types of ADCs.

[Figure 7 next here]

Because the cytotoxic drug linked to the mAb structure is quite lipophilic, its conjugation increases the hydrophobicity of the species, and therefore retention increases with the conjugation number. In most cases, a simple linear inverse salt gradient enables the separation of all the different DARs. In the literature, butyl phases have been mostly used to separate the DARs and gradients typically start with $1-1.5 \mathrm{M}$ ammonium sulfate $[9,78]$. The mobile phase $\mathrm{pH}$ is set between 6.5 and 7.0 by adding $10-100 \mathrm{mM}$ phosphate buffer. Other phase systems such as ether stationary phase with sodium chloride salt gradient were also successfully applied for DAR separations $[10,79]$.

From the HIC chromatographic profile, the average DAR can easily be calculated. Chromatograms have to be acquired at 214 or $280 \mathrm{~nm}$ (using UV detection) or at $\lambda_{\mathrm{ex}}$ : 280 $\mathrm{nm}, \lambda_{\mathrm{em}}: 360 \mathrm{~nm}$ (using fluorescence detection). Peak area percentages of the different species have to be determined. Then, the weighted peak areas have to be calculated by multiplying the peak area percentage by the corresponding drug load. Finally, weighted average DAR can be obtained by summing the weighted peak area percentages and dividing their sum by 100 [80]. The assignment of the HIC peak identity can be confirmed (in addition to elution order) by examining the UV spectra of the different peaks. The maximum absorbance of the mAb occur at $280 \mathrm{~nm}$, while the linker-drug portion has maximum UV absorbance at $248 \mathrm{~nm}$. When normalizing the spectra at $280 \mathrm{~nm}$, a proportional absorbance shift is observed at $248 \mathrm{~nm}$ [81]. Figure 8 shows the normalized UV spectra of HIC peaks of a cysteine linked ADC. 
[Figure 8 next here]

Obviously other techniques can also be used to determine the average DAR and DAR distribution [82]. UV-VIS spectrophotometry, RPLC or CGE-SDS are often used for cysteine linked ADCs, while IEX or cIEF are routinely used for lysine conjugations [78,82]. (Currently both cysteine and lysine conjugated ADCs are commercially available.) With RPLC, not only the average DAR but also the amount of positional isomers can be determined. Native MS is considered as an important tool for the determination of average DAR and DAR distribution [83]. The best way is to apply at least two different methods to confirm the average DAR.

\subsection{Determining the hydrophobicity of mAbs or ADCs}

In $\mathrm{HIC}$, the retention of proteins is mainly driven by hydrophobic interactions that occur between the stationary phase ligand and the amino acid moieties located on the surface of the protein. Under the usual conditions, secondary interactions are negligible. Therefore, proteins can be directly ranked, based on their hydrophobicity. Hydrophobicity of mAbs and related products (i.e. fusion proteins, ADCs) is an important characteristic as it correlates well with the aggregation propensity and stability. It is also useful for selecting the appropriate excipients to create a stable formulation.

A generic fast HIC method was currently developed in our laboratory to rank therapeutic mAbs on the basis of their hydrophobicity. A Tosoh TSK gel butyl-NPR column $(4.6 \times 35 \mathrm{~mm}$, $2.5 \mu \mathrm{m}$ ) was applied with mobile phase "A" of $2 \mathrm{M}$ ammonium sulfate and $0.1 \mathrm{M}$ phosphate buffer $(\mathrm{pH}=7)$ and mobile phase "B" of $0.1 \mathrm{M}$ phosphate buffer $(\mathrm{pH}=7)$. A gradient was performed from 40 to $70 \% \mathrm{~B}$ in 3 minutes at a flow rate of $1.2 \mathrm{~mL} / \mathrm{min}$. Method parameters were optimized to get apparent retention factors $\left(k_{\text {app }}\right)$ of all mAbs and related products between $\mathrm{k}_{\mathrm{app}}=1$ and $\mathrm{k}_{\mathrm{app}}=10$ within the possible shortest analysis time. Figure 9 shows the apparent retention factors (hydrophobicity ranking) of various commercial therapeutic mAbs and fusion proteins. During this study, biosimilar versions of some mAbs were also measured and showed some change in retention compared to the originator's product (data not shown).

[Figure 9 next here] 


\subsection{Determination of heterodimerization efficiency of bispecific antibodies}

BsAbs are artificial proteins that are composed of fragments of two different mAbs and therefore bind to two different types of antigens. BsAbs are expected to have biochemical characteristics intermediate to that of the corresponding parental antibodies (mAbA and mAbB) [84]. Hence, if the parental antibodies display significant differences in charge and/or hydrophobicity, the efficiency of the bsAbs can be ascertained by means of chromatographic techniques (IEX and HIC, respectively). HIC is commonly used for the determination of heterodimerization efficiency when the parental mAbs possess different hydrophobicity. As expected, the bsAb peak elutes between that of parental antibodies. Residual parental mAb content and the distribution of $\mathrm{mAbA}, \mathrm{mAbB}$ and $\mathrm{bsAb}$ species can be determined on the basis of HIC peak area percentages obtained at $280 \mathrm{~nm}$ [84]. Labrijn et. al. suggested recently a protocol for the analysis of Fab-arm exchange efficiency of bispecific IgG1 by HIC [85].

\section{Perspectives in HIC}

The current trends in liquid chromatography include the decrease of column dimensions (e.g. narrow-bore, micro-bore or capillary columns), decrease of particle sizes, change of particle morphology (superficially porous particles technology), increase of pressure capability or column coupling to perform high resolution separations [86]. Multidimensional separations is also attracting more and more attention for biopharmaceuticals characterization [87]. However, the technological progresses in HIC are relatively slow. This is probably due to the fact, that historically this mode was applied for proteins purification purposes. Now, HIC becomes increasingly important at the analytical scale, thanks to the strong developments of mAbs and ADCs, and therefore revolutionary progress in HIC column technology is expected in the close future.

\subsection{Decreasing particle size}


The Ultra-High Pressure Liquid Chromatography (UHPLC) technology was originally developed for RPLC applications, but columns packed with sub-3 $\mu \mathrm{m}$ and even sub-2 $\mu \mathrm{m}$ particles are also available for SEC [88] and IEX [89,90] operations.

Applying columns packed with sub-2 and sub-3 $\mu$ m particles may also open a new level of performance in $\mathrm{HIC}$, but it has to be kept in mind that with very fine particles, the separation quality is improved at the cost of pressure (and temperature gradients attributed to frictional heating effects) [91]. Therefore, there is a risk of on-column degradation and conformational changes when analyzing temperature or pressure sensitive proteins under high pressure (i.e., > 300 bar) conditions, as reported in RPLC and SEC [92,93]. From a commercial point of view, the smallest particle size in HIC currently available is $2.5 \mu \mathrm{m}$.

Our laboratory has tested a prototype $1.7 \mu \mathrm{m}$ non-porous butyl phases, but no obvious benefits were found compared to the existing materials packed with 2.5 or $3 \mu \mathrm{m}$ particles.

\subsection{Column coupling}

The efficiency (peak capacity) of gradient separations depends on several variables such as the gradient time, gradient steepness, flow rate, temperature and column length. One generic expression for peak capacity in gradient elution is the following one [94]:

$n_{c}=1+\frac{\sqrt{L}}{4 \sqrt{H}} \cdot \frac{1}{b+1} \cdot \ln \left(\frac{b+1}{b} e^{S \Delta \Phi}-\frac{1}{b}\right)$

with

$b=\frac{t_{0} \Delta \Phi S}{t_{g}}$

Where $L$ is the column length, $H$ is the plate height (similar as in the isocratic elution), $S$ is a gradient parameter (slope of the LSS equation, related to the solute nature, molecular weight and organic modifier nature), $\Delta \Phi$ is the change in solvent composition during the gradient, and $t_{0}$ is the column dead time. When maintaining the gradient steepness constant, the peak capacity is related to the square root of the column length. Therefore, to improve kinetic performance under a given gradient program, the column length has to be increased in 
agreement with the LSS theory and the geometrical scaling transfer rules [64,95]. This column coupling approach has already been applied for mAb separations in RPLC up to 45 cm column length [96]. Boyd et al. showed HIC separations of lgG1 oxidized samples, performed on $20 \mathrm{~cm}$ long column (two columns of $10 \mathrm{~cm}$ coupled in series) [81].

Figure 10 shows the theoretical impact of column length on ADC DAR separations in HIC mode. Model calculations were performed by assuming $100 \times 4.6 \mathrm{~mm}$ columns packed with 5 $\mu \mathrm{m}$ non-porous particles and coupling them to get 20 and $30 \mathrm{~cm}$ total length (L). A flow rate of $1 \mathrm{~mL} / \mathrm{min}$ flow rate was applied and a reverse salt gradient from 20 to $100 \% \mathrm{~B}$ (corresponds to $3 \mathrm{M}$ to $0 \mathrm{M}$ sodium chloride) was considered. The gradient time $\left(\mathrm{t}_{\mathrm{g}}\right)$ was adjusted in agreement with the column length, according to the geometrical transfer rules. Calculations are based on real initial experiments. As shown in Figure 10, the resolution (Rs) between the hydrophobic DAR species (DAR 6 and DAR 8) can be increased from $\mathrm{Rs}=1.14$ to 1.55 and 1.95 by increasing the column length from $10 \mathrm{~cm}$ to 20 and $30 \mathrm{~cm}$, respectively.

[Figure 10 next here]

\subsection{Two dimensional separations}

Multi-dimensional (2D) chromatography could be a powerful technique for mAb and ADC analysis and characterization, since it allows the hyphenation of non-compatible MS chromatographic method (i.e. HIC as the first dimension) to MS instrumentation via a suitable interface (involving the use of RPLC as the second dimension). Three approaches can be found in the literature applied for multi-dimensional separations of mAb and ADC samples, namely 1) off-line sample collection, 2) heart-cutting approach and 3) full comprehensive approach.

Debaene et al. applied HIC for off-line native MS characterization of an ADC, namely brentuximab vedotin [6]. HIC fractions were collected, desalted and then analyzed by native MS and ion mobility (IM) MS, assessing the interpretation of each HIC peak.

Applying on-line multi-dimensional LC facilitates the combination of HIC and MS using a RPLC desalting step prior to MS. Birdsall et al. showed the potential of an on-line HIC-RPLC- 
MS set-up for the characterization of isoforms of cysteine conjugated ADCs [97]. The ADCs dissociate into their respective sub-units under the denaturing reversed phase conditions (mobile phase and temperature). The heart-cutting setup provided unambiguous identification of positional isomers and the drug conjugation site confirmation. Although this approach was efficient, this 2D-LC method, - based on heart-cuts of the first dimension peaks - required as many injections as the number of peaks to be analyzed, which makes this strategy sample-consuming and hence less attractive when only a limited sample amount is available. Moreover, this approach may be time consuming and requires complex instrumentation (chromatographic setup with 3 pumps, including first and second dimension pumps as well as a regenerating pump). On-line comprehensive two-dimensional liquid chromatography could therefore be an attractive alternative for reducing both sample consumption and analysis time but remains a complex strategy. In addition, in on-line LCxLC, the entire first dimension separation is subjected to the second separation. As a result, the risk of missing peaks of interest which in turn may lead to lose essential information is avoided.

Recently an on-line HICxRPLC-MS method was successfully developed leading to an informative 2D-separation requiring $75 \mathrm{~min}$ only. The objective was to get relevant information on both drug loaded profile (average DAR) and structural information on positional isomers of each DAR of a cysteine-linked ADC (brentuximab vedotin, Adcetris ${ }^{\circledR}$ ) [98]. Figure 11 shows the optimized ${ }^{1} \mathrm{D}$ HIC separation and 2D-colour plots (UV-signal and Total lon Current MS signal) of the HIC x RPLC separation.

[Figure 11 next here]

\subsection{Online HIC-MS}

Recent progresses in top-down protein analysis has led to the demand for MS-compatible chromatographic techniques to separate intact proteins using volatile mobile phases. Conventional HIC provides "high-resolution" separation of proteins under non-denaturing conditions but requires high concentrations of nonvolatile salts. A recent study proposed a 
new series of more-hydrophobic HIC materials that can retain proteins using MS-compatible concentrations of ammonium acetate and $50 \%$ acetonitrile as organic modifier [99]. These new HIC materials appeared to work as a hybrid form of conventional HIC and RP chromatography (more hydrophobic than common HIC phases). The function of the salt seemed to be preserving protein structure rather than promoting retention. Online HIC-MS was feasible for both qualitative and quantitative analysis [99].

\section{Conclusion}

With the strong development of protein biopharmaceuticals (mainly mAbs and ADCs), HIC comes back on the forefront as an analytical technique to separate protein species based on their hydrophobicity. The main advantage of HIC over RPLC is its ability to perform separations under non denaturing conditions (i.e. physiological $\mathrm{pH}$ conditions, ambient mobile phase temperature and no need for organic solvents), which is particularly useful for ADCs characterization. To date, HIC has been used for several applications, including i) the determination of mAbs or ADCs hydrophobicity, 2) the determination of ADCs' average DAR and DAR distribution and 3) the monitoring of various post-translational modifications.

Numerous HIC stationary phases were commercialized over the last few years. These modern HIC phases are mostly based on silica or polymeric particles, and made of porous or non-porous material. Various chemistries are available to achieve reasonable retention for both hydrophilic and hydrophobic proteins. Columns are generally packed with particles of 2.5 to $5 \mu \mathrm{m}$ and some $1.7 \mu \mathrm{m}$ particles will certainly come to the market very soon, to compensate the poor kinetic performance of HIC.

As described in this review, the workflow for HIC method development can be improved, by using an initial screening procedure to find out the best phase system (stationary phase and salt nature/concentration), followed by a sound optimization of gradient conditions, temperature and $\mathrm{pH}$ using modelling software to build retention model and resolution map. The reliability of this optimization approach was found to be very good (less than $1 \%$ variation between predicted and experimental retention times). 
Finally, one of the very recent trend of HIC is the possibility to make this approach compatible with MS by using two-dimensional heart cutting or comprehensive approach involving HIC in the first dimension and RPLC in the second one. Based on the promising results achieved with this strategy, it is expected that more and more experiments will be performed using HICxRPLC-MS in the future.

\section{Acknowledgements}

Davy Guillarme and Jean-Luc Veuthey wish to thank the Swiss National Science Foundation for support through a fellowship to Szabolcs Fekete (31003A 159494). 


\section{References}

[1] A. Beck, T. Wurch, C. Bailly, N. Corvaia, Strategies and challenges for the next generation of therapeutic antibodies, Nat. Rev. Immunol. 10 (2010) 345-352.

[2] J. M. Reichert, A. Beck, A.A. Lugovskoy, T. Wurch, S. Coats, R.J. Brezski, 9th annual European Antibody Congress, November 11-13, 2013, Geneva, Switzerland, mAbs 6 (2014) 309-326.

[3] A. Beck, J.M. Reichert, Antibody-drug conjugates: present and future, mAbs 6 (2014) $15-17$.

[4] C. Klinguer-Hamour, P. Strop, D.K. Shah, L. Ducry, A. Xu, A. Beck, World Antibody-Drug Conjugate Summit, October 15-16, 2013, San Francisco, CA, mAbs 6 (2014) 18-29.

[5] R.V. Chari, Targeted cancer therapy: conferring specificity to cytotoxic drugs, Acc. Chem. Res. 41 (2008) 98-107.

[6] F. Debaene, A. Boeuf, E.W. Rousset, O. Colas, D. Ayoub, N. Corvaia, A.V. Dorsselaer, A. Beck, S. Cianférani, Innovative native MS methodologies for antibody drug conjugate characterization: high resolution native MS and IM-MS for average DAR and DAR distribution assessment, Anal. Chem., 86 (2014) 10674-10683.

[7] Q. Zhao, T.A. Stadheim, L. Chen, M.W. Washabaugh, in: Z. An (Eds.), Therapeutic Monoclonal Antibodies, John Wiley \& Sons, Inc. Hoboken, New Jersey, 2009, p. 525.

[8] A. Beck, H. Diemer, D. Ayoub, F. Debaene, E. Wagner-Rousset, C. Carapito, A. Van Dorsselaer, S. Sanglier-Cianferani, Analytical characterization of biosimilar antibodies and Fc-fusion proteins, Trends in Anal. Chem., 48 (2013) 81-95.

[9] B. Wiggins, L.L. Shin, H. Yamaguchi, G. Ratnaswamy, Characterization of cysteine-linked conjugation profiles of immunoglobin G1 and immunoglobin G2 antibody-drug-conjugates, J. Pharm. Sci., 104 (2015) 1362-1372.

[10] M. Haverick, S. Mengisen, M. Shameem, A. Ambrogelly, Separation of mAbs molecular variants by analytical hydrophobic interaction chromatography HPLC: overview and applications, mAbs 6 (2014) 852-858. 
[11] S. Hjerten, Some general aspects of hydrophobic interaction chromatography, J. Chromatogr. 87 (1973) 325-331.

[12] J.A. Querioz, C.T. Tomaz, J.M.S. Cabral, Hydrophobic interaction chromatography of proteins, J. Biotech., 87 (2001) 143-159.

[13] J.T. McCue, Theory and use of hydrophobic interaction chromatography in protein purification applications, Methods Enzymol. 463 (2009) 405-414.

[14] A. Vailaya, Cs. Horváth, Retention thermodynamics in hydrophobic interaction chromatography, Ind. Eng. Chem. Res. 35 (1996) 2964-2981.

[15] A. Mahn, Hydrophobic Interaction Chromatography: Fundamentals and Applications in Biomedical Engineering, chapter in Biomedical science, engineering and technology, ed. D.N. Ghista, intechweb.org, 2012.

[16] A. Tiselius, Adsorption separation by salting out, Mineral Geol., $26 \mathrm{~B}$ (1948) 1-5.

[17] S. Shalitel, Z. Er-el, Hydrophobic chromatography. Use for purification of glycogen synthetase, Proc. Natl. Acad. Sci. 70 (1973) 778-781.

[18] J. Porath, Salt-promoted adsorption: recent developments, J. Chromatogr. 376 (1986) 331-341.

[19] C. Tanford, The hydrophobic effect and the organization of living matter, Science 200 (1978) 1012-1018.

[20] C. Tanford, The hydrophobic effect: formation of micelles and biological membranes, Wiley-Interscience: New York, 1980; p 1.

[21] A. Ben-Naim, Hydrophobic Interactions, Plenum Press: New York, 1980.

[22] I. Molnár, Searching for robust HPLC methods - Csaba Horváth and the solvophobic theory, Chromatographia 62 (2005) S7-S17.

[23] S. Lewin, Displacement of water and its control of biochemical reactions. Academic Press, New York, 1974.

[24] H.P. Jennissen, Evidence of negative cooperavity in the adsorption of phosphorylase b on hydrophobic agaroses, Biochemistry, 15 (1976) 617-642.

[25] J.L. Ochoa, Hydrophobic (interaction) chromatography, Biochimie, 1978 (60) 1-15. 
[26] H.S. Frank, Free volume and entropy in condensed systems. II. Liquids, J. Chem. Phys. 13 (1945) 493-507.

[27] H.S. Frank, M.W. Evans, Free volume and entropy in condensed systems. III. Entropy in binary liquid mixtures; partial molal entropy in dilute solutions; structure and thermodynamics in aqueous electrolytes, J. Chem. Phys. 13 (1945) 507-532.

[28] J.M. Sturtevant, Heat capacity and entropy changes in processes involving proteins, Proc. Natl. Acad. Sci. U.S.A. 74 (1977) 2236-2240.

[29] J.R. Livingstone, R.S. Spolar, M.T. Record Jr., Contribution to the thermodynamics of protein folding from the reduction in water-accessible nonpolar surface area, Biochemistry 30 (1991) 4237-4244.

[30] R.L. Baldwin, Temperature dependence of the hydrophobic interaction in protein folding, Proc. Natl. Acad. Sci. U.S.A. 83 (1986) 8069-8072.

[31] Cs. Horváth, W. Melander, I. Molnár, Solvophobic interactions in liquid chromatography with non-polar stationary phases, J. Chromatogr. 125 (1976) 129-156.

[32] W. Melander, Cs. Horváth, Salt effects on hydrophobic interactions in precipitation and chromatography of proteins: an interpretation of the lyotrpic series, Arch. Biochem. Biophys. 183 (1977) 200-215.

[33] W. Melander, Cs. Horváth, Effect of neutral salts on the formation and dissociation of protein aggregates, J. Solid-Phase Biochem. 2 (1977) 141-161.

[34] N.T. Miller, B.L. Karger, High-performance hydrophobic-interaction chromatography on ether-bonded phases: Chromatographic characteristics and gradient optimization, J. Chromatogr. 326 (1985) 45-61.

[35] L. Szepesy, Cs. Horváth, Specific salt effects in hydrophobic interaction chromatography of proteins, Chromatographia 26 (1988) 13-18.

[36] T. Arakawa, S.N. Timasheff, Mechanism of protein salting in and salting out by divalent cation salts: balance between hydration and salt binding, Biochemistry 23 (1984) 5912-5923. [37] H. Inoue, S.N. Timasheff, Preferential and absolute interactions of solvent components with proteins in mixed solvent systems, Biopolymers 11 (1972) 737-743. 
[38] K. Gekko, S.N. Timasheff, Mechanism of protein stabilization by glycerol: preferential hydration in glycerol-water mixtures, Biochemistry 20 (1981) 4667-4676.

[39] T. Arakawa, S.N. Timasheff, Preferential interactions of proteins with solvent components in aqueous amino acid solutions, Arch. Biochem. Biophys. 224 (1983) 169-177. [40] T. Arakawa, S.N. Timasheff, Mechanism of poly(ethylene glycol) interaction with proteins, Biochemistry 24 (1985) 6756-6762.

[41] T. Arakawa, S.N. Timasheff, Abnormal solubility behavior of beta-lactoglobulin: salting-in by glycine and $\mathrm{NaCl}$, Biochemistry 26 (1987) 5147-5153.

[42] G. Rippel, Á. Bede, L. Szepesy, Systematic method development in hydrophobic interaction chromatography I. Characterization of the phase system and modelling retention, J. Chromatogr. A, 697 (1995) 17-29.

[43] M.R. Rodriguez, D. Guillarme, A. Beck, S. Fekete, Practical method development for the separation of monoclonal antibodies and antibody-drug-conjugate species in hydrophobic interaction chromatography, Part 1: Optimization of the mobile phase, J. Pharm. Biomed. Anal. 118 (2016) 393-403.

[44] A. Cusumano, D. Guillarme, A. Beck, S. Fekete, Practical method development for the separation of monoclonal antibodies and antibody-drug-conjugate species in hydrophobic interaction chromatography, Part 2: Optimization of the phase system, J. Pharm. Biomed. Anal. 121 (2016) 161-173.

[45] S. Hjertén, J. Rosengren, S. Pahlman, Hydrophobic interaction chromatography, J. Chromatogr. 101 (1974) 281-288.

[46] V. Ulbrich, J. Makes, M. Jurecek, Identification of giycidyl ethers. Bis(phenyl-)and bis(anaphthylurethans) of glycerol a-alkyl (aryl)ethers, Collect. Czech. Chem. Commun. 29 (1964) 1466-1475.

[47] J.L. Fausnaugh, L.A. Kennedy, F.E. Regnier, Comparison of hydrophobic-interaction and reversed-phase chromatography of proteins, J. Chromatogr. 317 (1984) 141-155.

[48] F.Y. Lin, W.Y. Chen, R.C. Ruaan, H.M. Huang, Microcalorimetric studies of the interactions between proteins and hydrophobic ligands in hydrophobic interaction 
chromatography: effects of chain length, density and the amount of bound protein. J. Chromatogr. A 872 (2000) 37-47.

[49] J. Porath, B. Larsson, Charge-transfer and water-mediated chromatography. I. Electronacceptor ligands on cross-linked dextran. J. Chromatogr. 155 (1978) 47-68.

[50] S. Fekete, J.L. Veuthey, D. Guillarme, Modern column technologies for the analytical characterization of biopharmaceuticals in various liquid chromatographic modes, LC GC Eur, Suppl.: S (2015) 8-15.

[51] S. Páhlman, J. Rosengren, S. Hjertén, Hydrophobic interaction chromatography on uncharged Sepharose derivatives. Effects of neutral salts on the adsorption of proteins, J. Chromatogr. 131 (1977) 99-108.

[52] G. Rippel and L. Szepesy, Hydrophobic interaction chromatography of proteins on an Alkyl-Superose column, J. Chromatogr. A, 664 (1994) 27-32.

[53] L. Szepesy and G. Rippel, Comparison and evaluation of HIC columns of different hydrophobicity, Chromatographia, 34 (1992) 391-397.

[54] L. Szepesy and G. Rippel, Effect of the characteristics of the phase system on the retention of proteins in hydrophobic interaction, J. Chromatogr. A, 668 (1994) 337-344.

[55] S.E. Builder, Hydrophobic interaction chromatography, principles and methods, Amersham pharmacia biotech, ISBN 91-970490-4-2, edition AB, 1993.

[56] D. Ekeberg, K.S. Gretland, J. Gustafsson, S.M. Braten, G.E. Fredheim, Characterisation of lignosulphonates and kraft lignin by hydrophobic interaction chromatography, Anal. Chim. Acta, 565 (2006) 121-128.

[57] J. Porath, L. Sundberg, N. Fornstedt, I. Olson, Salting-out in amphiphilic gels as a new approach to hydrophobic adsorption, nature 245 (1973) 465-466.

[58] G. Halperin, M. Breitenbach, M. Tauber-Finkelstein, S. Shaltiel, Hydrophobic chromatography on homologous series of alkyl agaroses. A comparison of charged and electrically neutral column materials, J. Chromatogr. 215 (1981) 211-228. 
[59] S. Hjertén, K. Yao, K.O. Eriksson, B. Johansson, Gradient and isocratic high performance hydrophobic interaction chromatography of proteins on agarose columns, J. Chromatogr. 359 (1986) 99-109.

[60] S.L. Wu, K. Benedek, B.L. Karger, Thermal behavior of proteins in high-performance hydrophobic-interaction chromatography. On-line spectroscopic and chromatographic characterization, J. Chromatogr. 359 (1986) 3-17.

[61] S.L. Wu, A. Figueroa, B.L. Karger, Protein conformational effects in hydrophobic interaction chromatography. Retention characterization and the role of mobile phase additives and stationary phase hydrophobicity, J. Chromatogr. 371 (1986) 3-27.

[62] D. Haidacher, A. Vailaya, Cs. Horváth, Temperature Effects in Hydrophobic Interaction Chromatography, Proc. Natl. Acad. Sci. U.S.A. 93 (1996) 2290-2295.

[63] L.R. Snyder, J.W. Dolan, High-performance gradient elution: The practical application of the Linear-Solvent-Strength model, 2007. John Wiley \& Sons, Inc., Hoboken, New Jersey, USA

[64] L.R. Snyder, J.J. Kirkland, J.L. Glajch, Practical HPLC Method Development, second ed., John Wiley \& Sons Inc., 1997.

[65] J. R. Montano, C.O. Bolsico, M.J. R. Angel, M.C.G.A. Coque, Implementation of gradients of organic solvent in micellar liquid chromatography using DryLab ${ }^{\circledR}$ : Separation of basic compounds in urine samples, J. Chromatogr. A 1344 (2014) 31-41.

[66] I. Molnar, Computerized design of separation strategies by reversed-phase liquid chromatography: development of DryLab software, J. Chromatogr. A 965 (2002) 175-194.

[67] S. Bhuvanesh, C. Arunkumar, P. Kaliraj, S. Ramalingam, S. Production and single step purification of Brugia malayi abundant larval transcript (ALT-2) using hydrophobic interaction chromatography, J. Ind. Micr.Biotech. 37 (2010) 1053-1059.

[68] C.B. Lavery, M.C. Maclnnis, M.J. MacDonald, J.B. Williams, C.A. Spencer, A.A. Burke, D.J. Irwin, G.B. D'Cunha, Purification of peroxidase from Horseradish (Armoracia rusticana) roots, J. Agric. Food Chem. 58 (2010) 8471-8476. 
[69] B. Liu, L. Huang, H. Buchenauer, Z. Kang, Isolation and partial characterization of an antifungal protein from the endophytic Bacillus subtilis strain EDR4, Pestic. Biochem. Phys. 98 (2010) 305-311.

[70] Y. Teng, Q. Yin, M. Ding, F. Zhao, Purification and characterization of a novel endobeta-1,4-glucanase, AfEG22, from the giant snail, Achatina fulica frussac. Acta Biochim. Biophys. Sin., 42 (2010) 729-734.

[71] D. Boyd, T. Kaschak, B. Ya, HIC resolution of an $\lg G 1$ with an oxidized Trp in a complementarity determining region, J. Chromatogr. B, 879 (2011) 955-960.

[72] J.V. Douglass, A. Wallace, A. Balland, Separation of populations of antibody variants by fine tuning of hydrophobic-interaction chromatography operating conditions, J. Chromatogr. A, 1214 (2008) 81-89.

[73] R.J. Harris, Heterogeneity of recombinant antibodies: linking structure to function, Dev. Biol. (Basel), 122 (2005) 117-127.

[74] US patent Enbrel 7,294,481 B1.

[75] B.Q. Shen, K. Xu, L. Liu, H. Raab, S. Bhakta, M. Kenrick, K.L. Parsons-Reponte, J. Tien, S.F. Yu, E. Mai, D. Li, J. Tibbitts, J. Baudys, O.M. Saad, S.J. Scales, P.J. McDonald, P.E. Hass, C. Eigenbrot, T. Nguyen, W.A. Solis, R.N. Fuji, K.M. Flagella, D. Patel, S.D. Spencer, L.A. Khawli, A. Ebens, W.L. Wong, R. Vandlen, S. Kaur, M.X. Sliwkowski, R.H. Scheller, P. Polakis, J.R. Junutula, Conjugation site modulates the in vivo stability and therapeutic activity of antibody-drug conjugates. Nat. Biotechnol. 30 (2012)184-189.

[76] Y.T. Adem, K.A. Schwarz, E. Duenas, T.W. Patapoff, W.J. Galush, O. Esue, Auristatin antibody drug conjugate physical instability and the role of drug payload. Bioconjug. Chem. 25 (2014) 656-664.

[77] B. Bender, D.D. Leipold, K. Xu, B.Q. Shen, J. Tibbitts, L.E. Friberg, A mechanistic pharmacokinetic model elucidating the disposition of trastuzumab emtansine (T-DM1), an antibody-drug conjugate (ADC) for treatment of metastatic breast cancer. AAPS. J. 16 (2014) 994-1008. 
[78] L.N. Le, J.M. Moore, J. Ouyang, X. Chen, M.D. Nguyen, W.J. Galush, Profiling antibody drug conjugate positional isomers: A system of-equations approach. Anal. Chem. 84 (2012) 7479-7486.

[79] T. Chen, K. Zhang, J. Gruenhagen, C.D. Medley, Hydrophobic interaction chromatography for antibody drug conjugate drug distribution analysis, Am. Pharm. Rev. (2015)

[80] J. Ouyang, Drug-to-antibody ratio (DAR) and drug load distribution by hydrophobic interaction chromatography and reversed phase high-performance liquid chromatography, chapter 17 in Antibody Drug Conjugates, Ed. L. Ducry, Book Methods in Molecular Biology 1045 (2013)

[81] K.J. Hamblett, P.D. Senter, D.F. Chace, M.M. Sun, J. Lenox, C.G. Cerveny, K.M. Kissler, S.X. Bernhardt, A.K. Kopcha, R.F. Zabinski, D.L. Meyer, J.A. Francisco, Effects of drug loading on the antitumor activity of a monoclonal antibody drug conjugate, Clin Cancer Res 10 (2004) 7063-7070.

[82] A. Wakankar, Y. Chen, Y. Gokarn, F.S. Jacobson, Analytical methods for physicochemical characterization of antibody drug conjugates, mAbs 3 (2011) 161-172.

[83] A. Beck, G. Terral, F. Debaene, E. Wagner-Rousset, J. Marcoux, M.C. Janin-Bussat, O. Colas, A. Van Dorsselaer, S. Cianférani, Cutting-edge mass spectrometry methods for the multi-level structural characterization of antibody-drug conjugates, Exp. Rev. Prot., 13 (2016) 157-183.

[84] S. Paul, J. Connor, T. Nesspor, P. Haytko, K. Boakye, M.L. Chiu, H. Jiang, An efficient process of generating bispecific antibodies via controlled Fab-arm exchange using culture supernatants, Exp. Rev. Prot., 121 (2016) 133-140.

[85] A.F. Labrijn, J.I. Meesters, P. Priem, R.N. de Jong, E.T.J. van den Bremer, M.D. van Kampen, A.F. Gerritsen, J. Schuurman, P.W.H.I. Parren, Controlled Fab-arm exchange for the generation of stable bispecific IgG1, Nature Protocols, 9 (2014) 2450-2463.

[86] S. Fekete, A.G.G. Perrenoud, D. Guillarme, Evolution and current trends in liquid and supercritical fluid chromatography, Curr. Chromatogr., 1 (2014) 15-40. 
[87] K. Sandra, P. Sandra, The opportunities of 2D-LC in the analysis of monoclonal antibodies, Bioanalysis, 7 (2015) 2843-2847.

[88] S. Fekete, A. Beck, J.L. Veuthey, D. Guillarme, Theory and practice of size exclusion chromatography for the analysis of protein aggregates, J. Pharm. Biomed. Anal., 101 (2014) 161-173.

[89] Agilent Technologies Inc., Agilent Bio MAb columns, (2010) Data sheet: 5973-1744

[90] Sepax Technologies, Cation exchange chromatography, (2012) Application note: 1-877SEPAX-US

[91] S. Fekete, D. Guillarme, Estimation of pressure-, temperature- and frictional heatingrelatedeffects on proteins' retention under ultra-high-pressure liquidchromatographic conditions, J. Chromatogr. A, 1393 (2015) 73-80.

[92] S. Fekete, S. Rudaz, J.L. Veuthey, D. Guillarme, Impact of mobile phase temperature on recovery and stability of monoclonal antibodies using recent reversed-phase stationary phases, J. Sep. Sci., 35 (2012) 3113-3123.

[93] S. Fekete, K. Ganzler, D. Guillarme, Critical evaluation of fast size exclusion chromatographic separations of protein aggregates, applying sub-2 $\mu \mathrm{m}$ particles, J. Pharm. Biomed. Anal., 78-79 (2013) 141-149.

[94] U.D. Neue, Theory of peak capacity in gradient elution, J. Chromatogr. A 1079 (2005) 153-161.

[95] L.R. Snyder, Gradient elution. In HPLC: Advances and Perspectives, Vol. 1 (C. Horvath, ed.) Academic Press, New York. 1980, pp. 208-316.

[96] S. Fekete, M.W. Dong, T. Zhang, D. Guillarme, High resolution reversed phase analysis of recombinant monoclonal antibodies by ultra-high pressure liquid chromatography column coupling, J. Pharm. Biomed. Anal., 83 (2013) 273-278.

[97] R. Birdsall, H. Shion, F.W. Kotch, A. Xu, T.J. Porter, W. Chen, A rapid on-line method for mass spectrometric confirmation of a cysteine-conjugated antibody-drug-conjugate structure using multidimensional chromatography, mAbs 7 (2015) 1036-1044. 
[98] M. Sarrut, A. Corgier, S. Fekete, D. Guillarme, D. Lascoux, M.C. Janin-Bussat, A. Beck, S. Heinisch, Analysis of antibody-drug conjugates by comprehensive on-line two-dimensional hydrophobic interaction chromatography $x$ reversed phase liquid chromatography hyphenated to high resolution mass spectrometry I - Optimization of conditions, J. Chromatogr. B, submitted (2016)

[99] B. Chen, Y. Peng, S.G. Valeja, L. Xiu, A.J. Alpert, Y. Ge, Online hydrophobic interaction chromatography - mass spectrometry for top-down proteomics, Anal. Chem. 88 (2016) 18851891. 


\section{Figure captions}

Figure 1. Analyzing intact and reduced mAb (adalimumab) in RPLC and HIC. The light- and heavy-chain ( $L$ and $H$ ) can be separated in generic RP conditions while the reduced antibody maintains its physiological " $Y$ " shape in generic HIC conditions. Data taken from the authors' laboratory. HIC conditions: MabPac RP $100 \mathrm{~mm}$ x $2.1 \mathrm{~mm}$ column, mobile phase "A": $0.1 \%$ TFA, mobile phase "B": $0.1 \%$ TFA in acetonitrile, 25 - $40 \%$ B gradient in 8 min, flow rate: $0.4 \mathrm{~mL} / \mathrm{min}$, temperature: $80^{\circ} \mathrm{C}$. RP conditions: MabPac HIC-10 $100 \mathrm{~mm}$ x $4.6 \mathrm{~mm}$ column, mobile phase "A": $2 \mathrm{M}$ ammonium-sulfate $+0.1 \mathrm{M}$ phosphate $(\mathrm{pH}=7)$, mobile phase "B": 0.1 M phosphate $(\mathrm{pH}=7), 0-100 \% \mathrm{~B}$ gradient in $10 \mathrm{~min}$, flow rate: $1 \mathrm{~mL} / \mathrm{min}$, temperature: 25 ${ }^{\circ} \mathrm{C}$.

Figure 2. Schematic view of protein retention mechanism in HIC. (with permission from Ref [13])

Figure 3. Impact of stationary phase on retention and selectivity. Separation of monoclonal antibody mixture [denosumab (1), palivizumab (2), pertuzumab (3), rituximab (4), bevacizumab (5)], on different stationary phases $(100 \times 4.6 \mathrm{~mm})$ using the same mobile phase, gradient, flow rate and detection. Data taken from the authors' laboratory.

Figure 4. Impact of organic modifier on retention and selectivity. Separation of monoclonal antibody mixture [denosumab (1), palivizumab (2), pertuzumab (3), rituximab (4), bevacizumab (5)], using a generic gradient from 2 to $0 \mathrm{M}$ ammonium sulfate on a $100 \times 4.6$ mm column. The mobile phase also contained $0.1 \mathrm{M}$ phosphate buffer $(\mathrm{pH}=7)$, and 0,7 and $14 \%$ isopropanol (IPA) was added to mobile phase "B". Temperature was set at $25^{\circ} \mathrm{C}$. Data taken from the authors' laboratory. 
Figure 5. Proposed workflow of HIC method development for the separation of mAbs and related products (ADCs). Mobile phase "A" contains salt (high concentration) and buffer (low concentration), mobile phase "B" contains buffer (low concentration).

Figure 6. HIC separation of modified mAb species (Asp $\rightarrow$ iso-Asp) developed during storage at $40{ }^{\circ} \mathrm{C}$ for 12 weeks. (with permission from Ref [72])

Figure 7. Possible DAR species and positional isomers of $\lg \mathrm{G} 1$ and $\lg \mathrm{G} 2$ based ADCs (A), and typical HIC profile of IgG1 based (B) and IgG2 based cysteine linked ADCs. D0 - D12 refers to different DAR species. (with permission from Ref [9])

Figure 8. Normalized UV spectra of drug loaded species (HIC peaks) of a cysteine linked lgG1 type ADC. (with permission from Ref [80])

Figure 9. Ranking of mAbs and fusion proteins on the basis of their hydrophobicity (apparent retention factor), using a generic HIC method. Conditions are detailed in section 4.3.

Figure 10. The impact of column length on the resolution of ADC DAR species. Calculations were performed assuming $100 \times 4.6 \mathrm{~mm}$ columns packed with $5 \mu \mathrm{m}$ non-porous particles operated at $1 \mathrm{~mL} / \mathrm{min}$ flow rate by developing an inverse salt gradient from 20 to $100 \% \mathrm{~B}$ (corresponds to $3 \mathrm{M}$ to $0 \mathrm{M}$ sodium chloride). Chromatogram predictions were done by DryLab modeling software.

Figure 11. optimized ${ }^{1} \mathrm{D}$ HIC separation and 2D-colour plots (UV-signal and Total lon Current MS signal) of the HIC x RPLC separation of brentuximab-vedotin sample. (with permission from Ref [98]) 

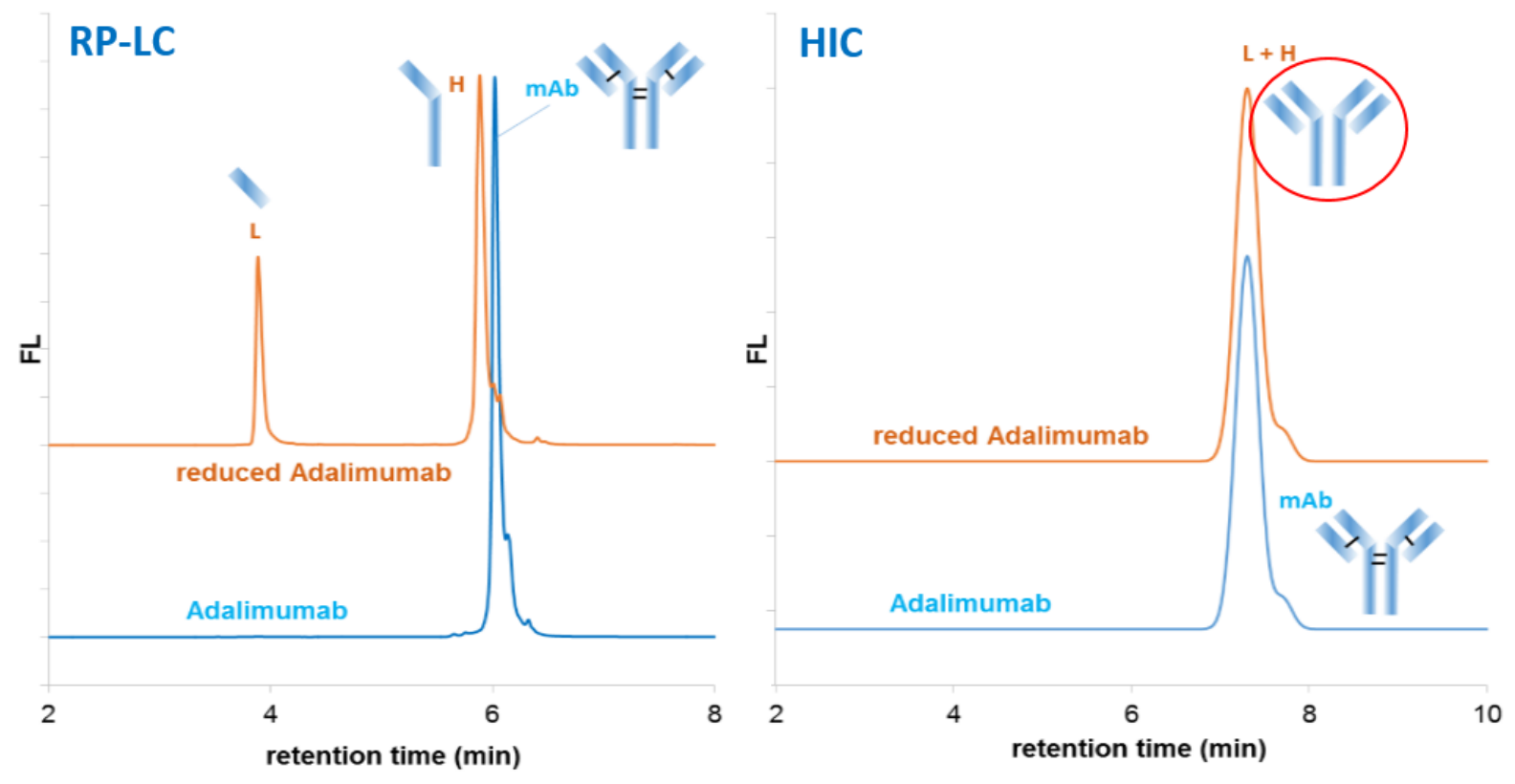

Figure 1. 

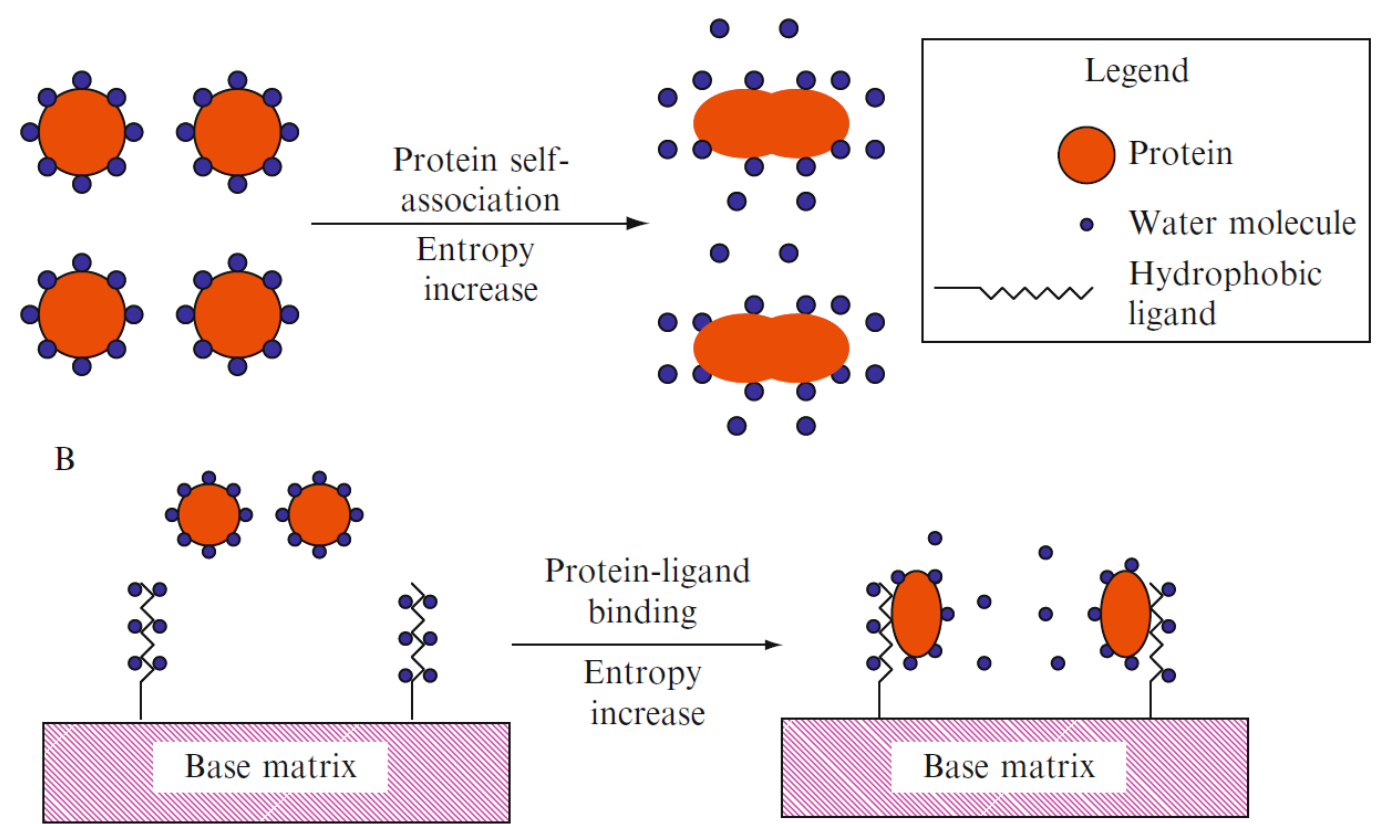

Figure 2. 


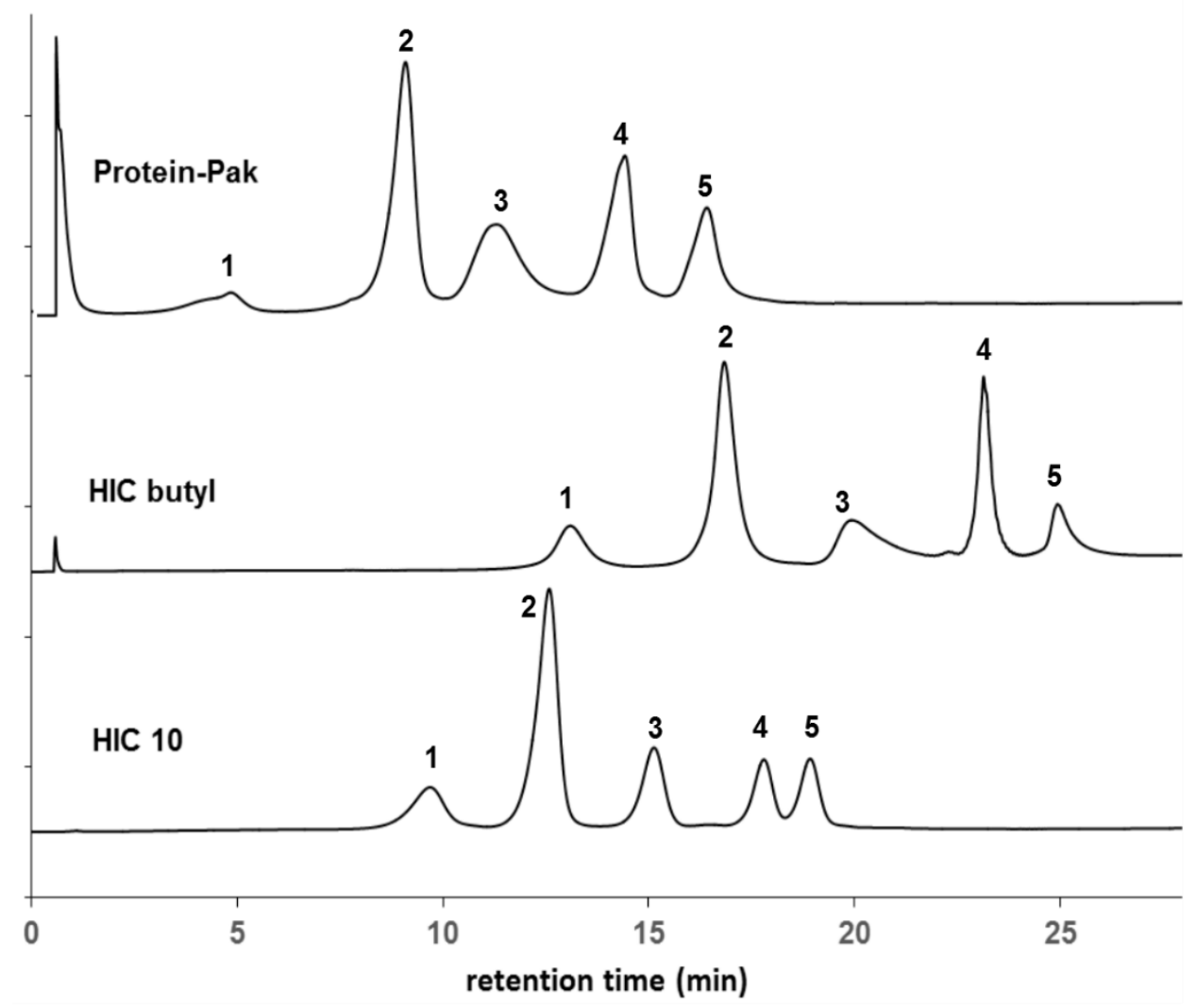

Figure 3. 

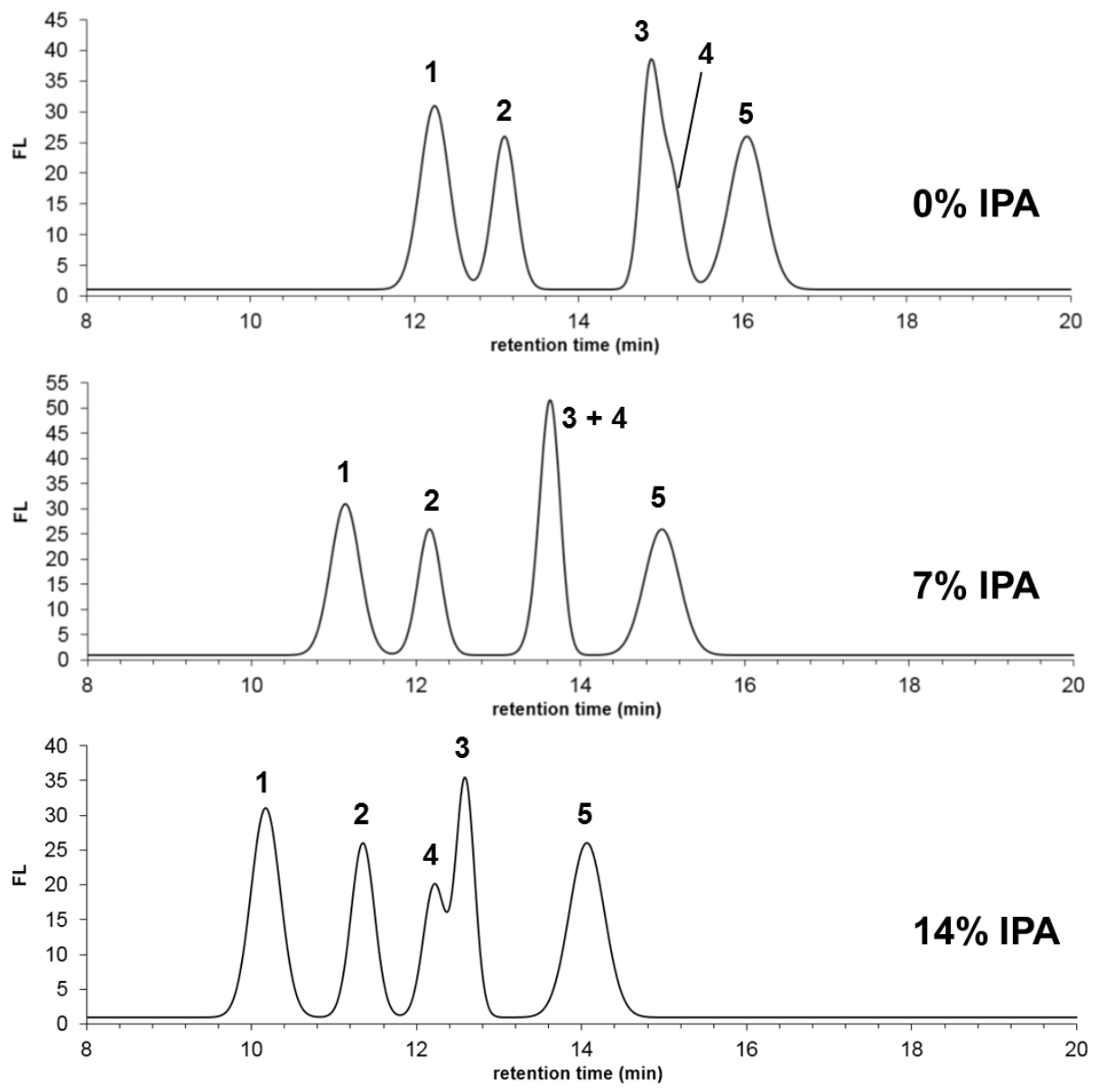

Figure 4. 


\section{Step 1: Finding the phase system}

Scouting gradient experiments on three columns (of different hydrophobicity) with different salt systems

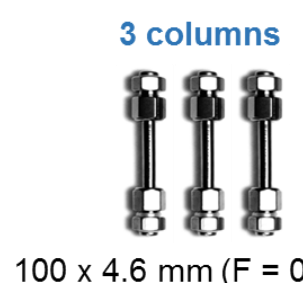

X 3 salts (e.g.):

$100 \times 4.6 \mathrm{~mm}(\mathrm{~F}=0.6 \mathrm{~mL} / \mathrm{min})$

1.5 $\mathrm{M}$ ammonium sulfate

3.3 $\mathrm{M}$ sodium acetate

3.9 $\mathrm{M}$ sodium chloride

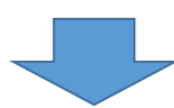

Provide roughly equivalent lyotropic strength on most HIC phases for mAbs and ADC species.

Selection of the appropriate phase system (one column and one salt) that provides sufficient retention and elution window for the peaks of interest

Step 2: Optimization of gradient program and other variables (temperature, organic modifier)

Performing initial runs based on one of the following experimental designs
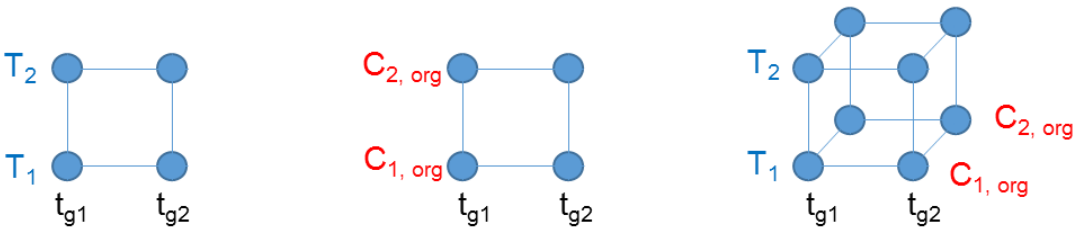

$$
\begin{aligned}
& \mathrm{t}_{\mathrm{g} 1}=10 \mathrm{~min} \\
& \mathrm{t}_{\mathrm{g} 2}=30 \mathrm{~min} \\
& \mathrm{~T}_{1}=20^{\circ} \mathrm{C} \\
& \mathrm{T}_{2}=40^{\circ} \mathrm{C} \\
& \mathrm{c}_{1, \text { org }}=0 \% \\
& \mathrm{c}_{2, \text { org }}=10 \%
\end{aligned}
$$

Peak tracking, building the retention model and resolution map

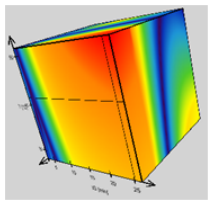

$$
\underset{\text { and analysis time }}{\stackrel{\text { Optimizing resolution }}{\longrightarrow}}
$$

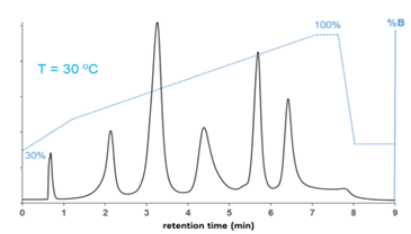

\section{Figure 5.}




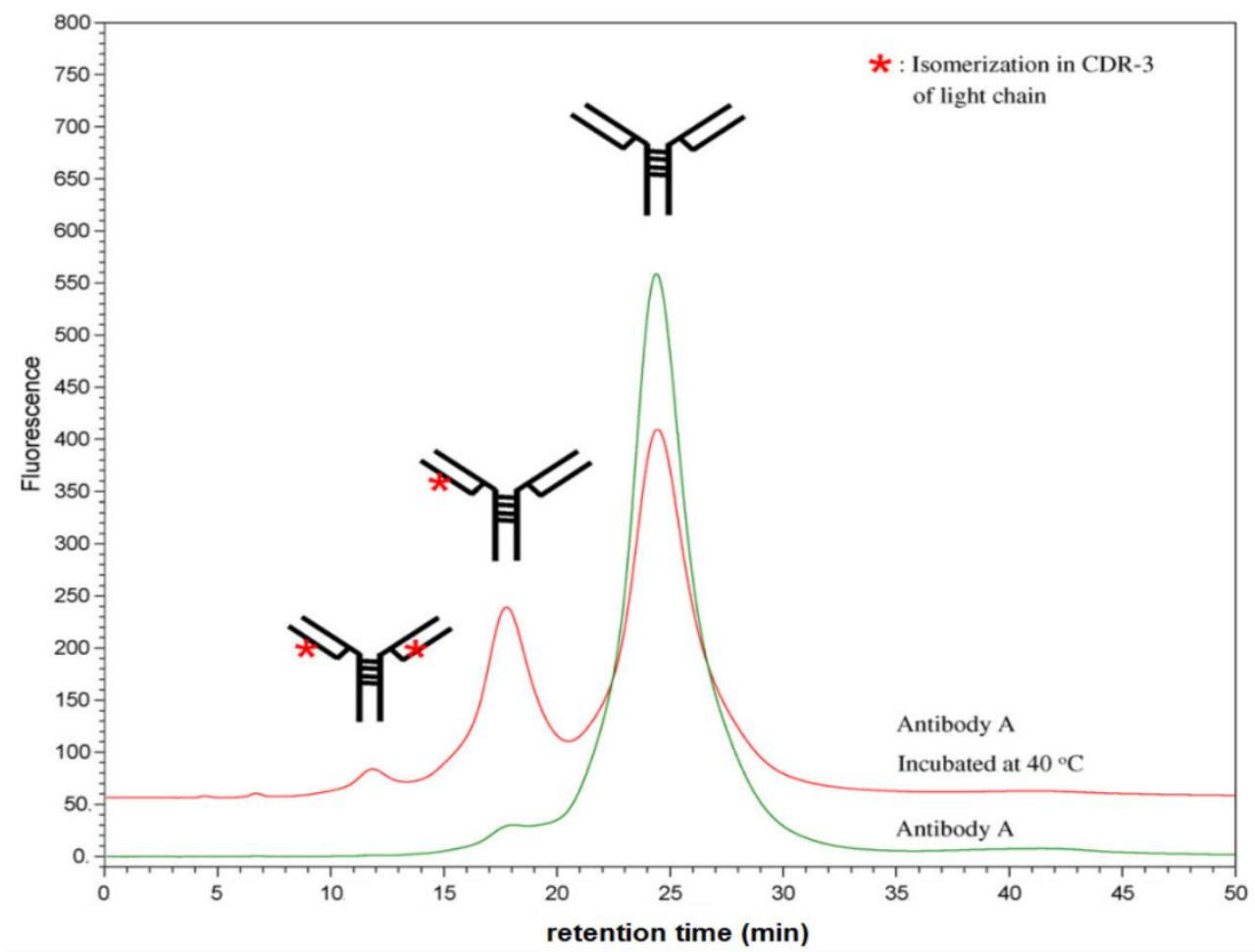

Figure 6. 
(A)

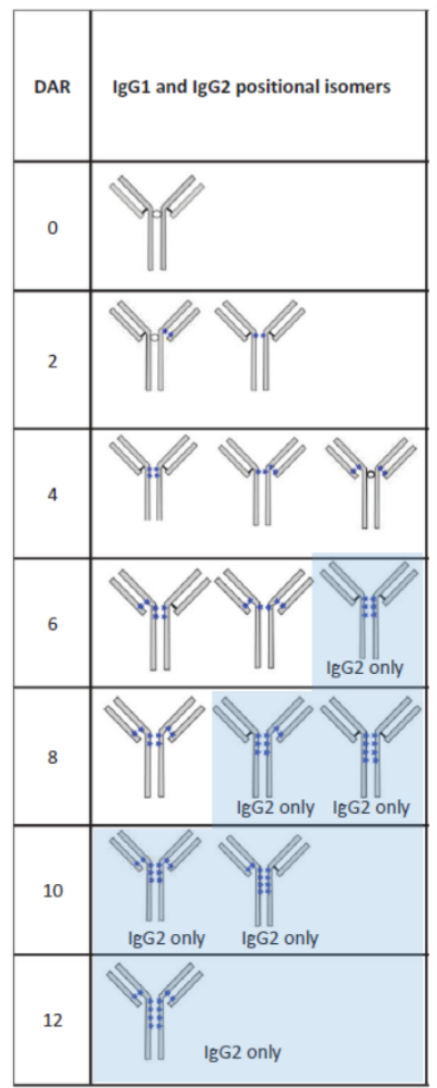

(B)

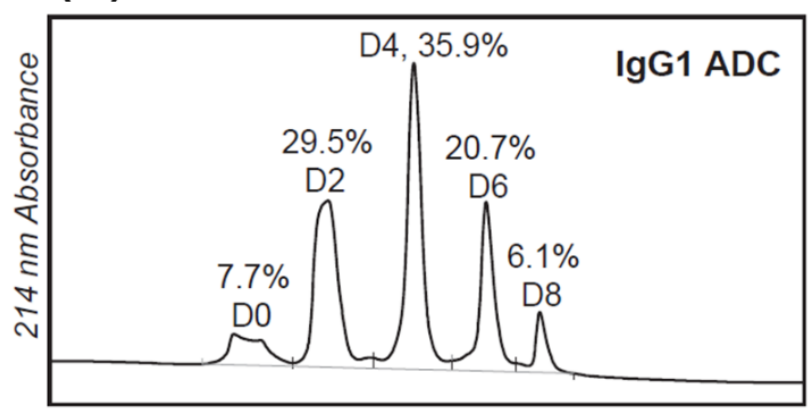

(C)

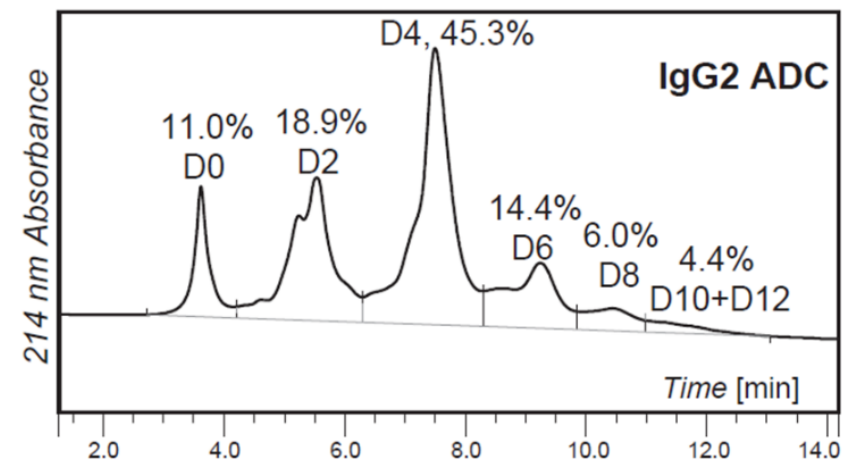

Figure 7. 


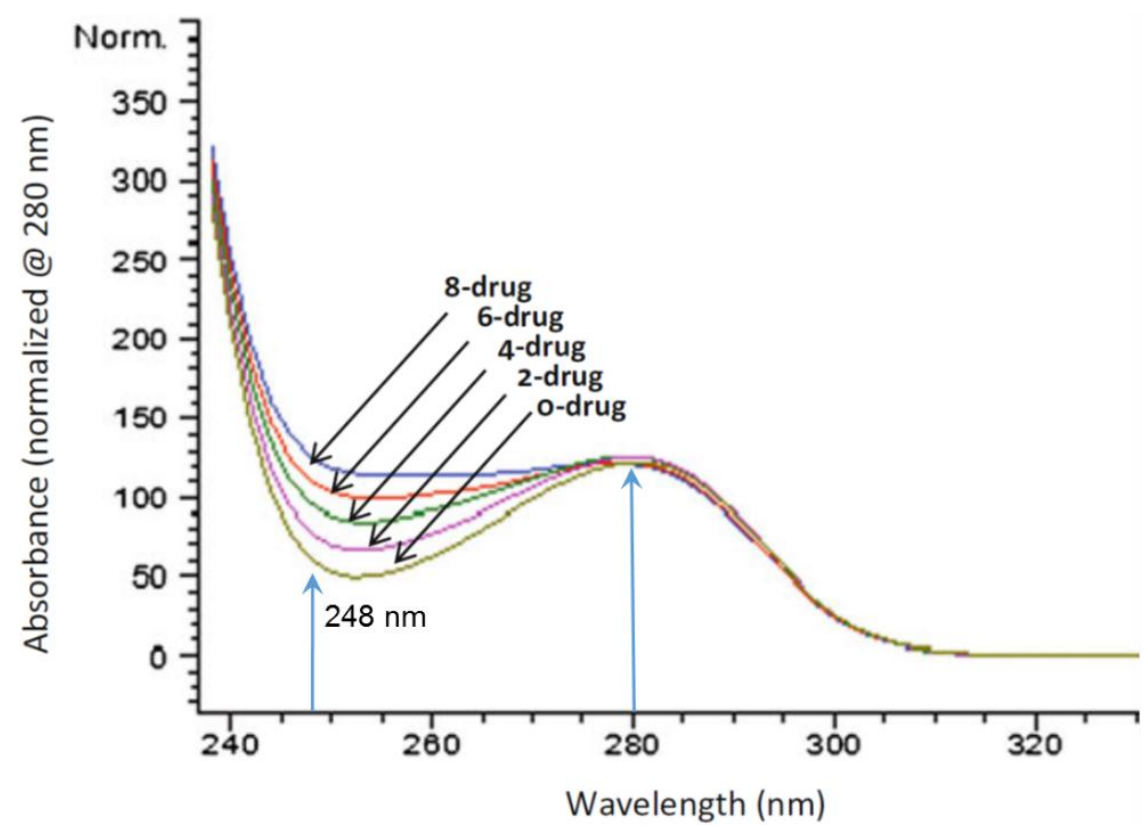

Figure 8. 


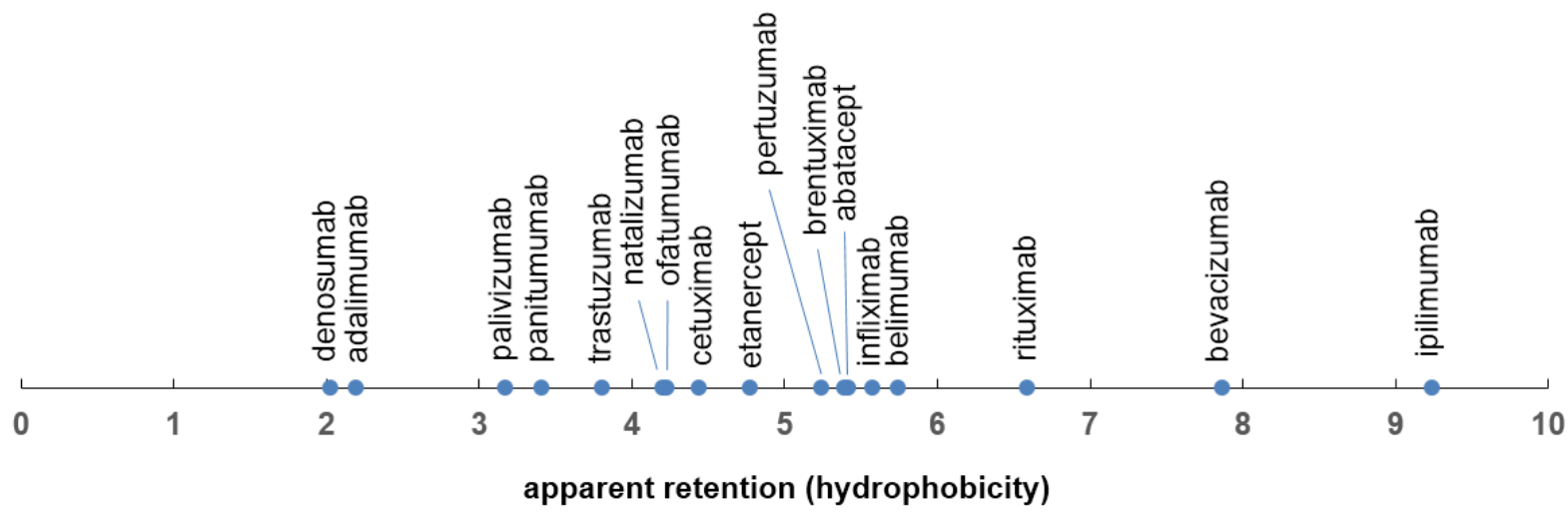

Figure 9. 


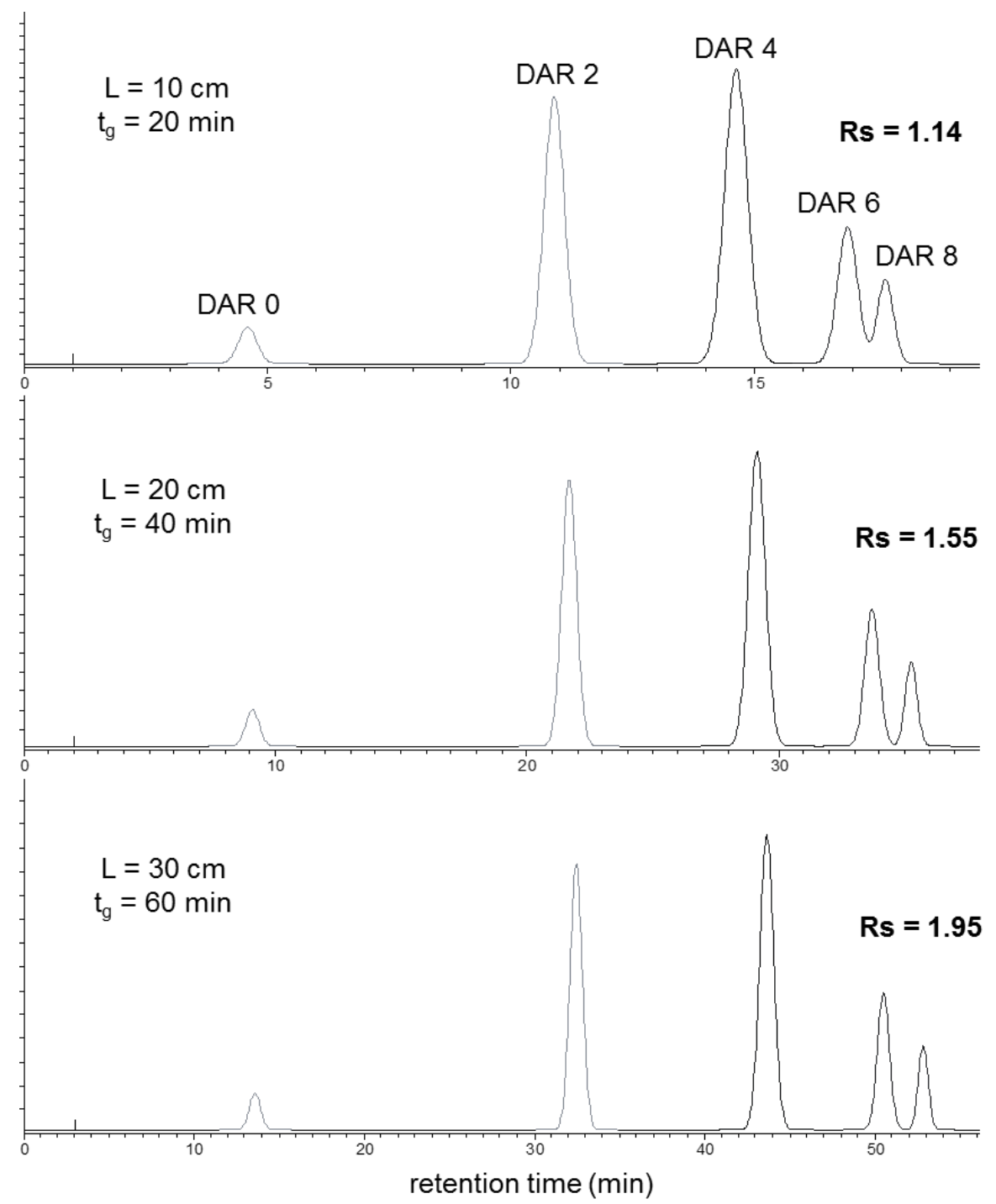

Figure 10. 

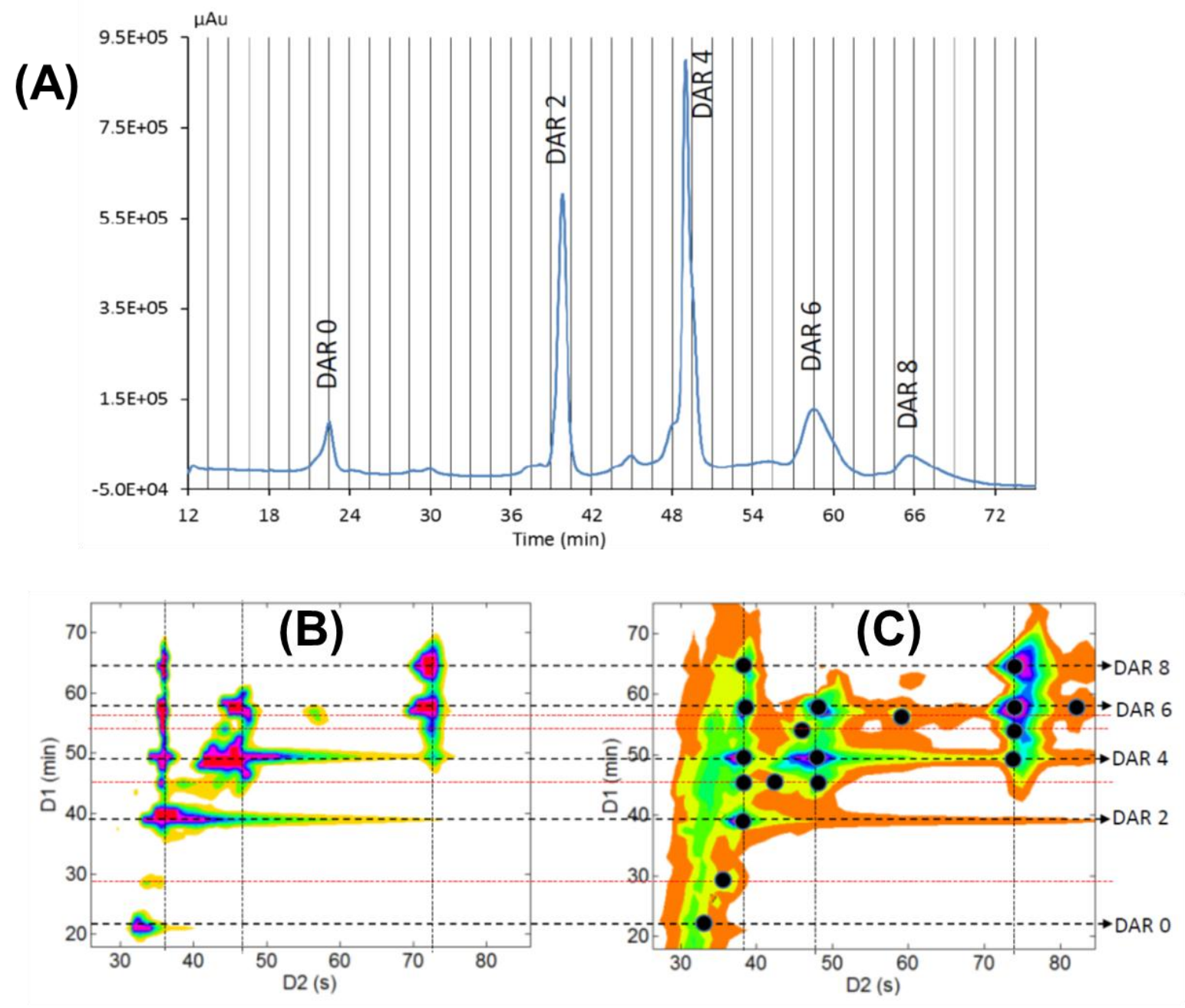

Figure 11. 